\title{
Entanglement-assisted capacities of compound quantum channels
}

\author{
Mario Berta, Hrant Gharibyan, and Michael Walter
}

\begin{abstract}
We study universal quantum codes for entanglementassisted quantum communication over compound quantum channels. In this setting, sender and receiver do not know the specific channel that will be used for communication, but only know the set that the channel is selected from. We investigate different variations of the problem: uninformed users, informed receiver, informed sender, and feedback assistance. We derive single-letter formulas for all corresponding channel capacities. Our proofs are based on one-shot decoupling bounds and properties of smooth entropies.
\end{abstract}

Index Terms-Quantum Mechanics, Quantum Entanglement, Compound Channels, Decoupling.

\section{INTRODUCTION}

$\mathbf{S}$ HANNON'S noisy channel coding theorem establishes that the asymptotic capacity of a fixed independent and identically distributed (IID) channel $\mathcal{J}_{X \rightarrow Y}$ is given by the mutual information between the input and the output of the channel [1],

$$
C\left(\mathcal{J}_{X \rightarrow Y}\right)=\sup _{P} I(X: Y) \mathcal{J}(P),
$$

optimized over all channel inputs. Moreover, it is known that the capacity is neither increased by feedback assistance from the receiver to the sender nor by shared randomness assistance between the sender and the receiver [1]. ${ }^{1}$ Extending Shannon's seminal result (1), there has been lots of work concerning scenarios where the channel is not fully known but there is some channel uncertainty, see, e.g., the review article [4]. Here, we are interested in the so-called compound setting when the sender and receiver do not know the specific channel that will be used for communication, but only know the set that the channel is selected from. For compound channels $\Lambda_{X \rightarrow Y}=$

M. Berta acknowledges funding by the SNSF through a fellowship, funding by the Institute for Quantum Information and Matter (IQIM), an NSF Physics Frontiers Center (NFS Grant PHY-1125565) with support of the Gordon and Betty Moore Foundation (GBMF-12500028), and funding support form the ARO grant for Research on Quantum Algorithms at the IQIM (W911NF-121-0521). H. Gharibyan and M. Walter gratefully acknowledge support from FQXI and the Simons Foundation. M. Walter also gratefully acknowledges support from AFOSR grant FA9550-16-1-0082.

M. Berta is with the Institute for Quantum Information and Matter California Institute of Technology, Pasadena, CA 91125 USA (e-mail: berta@caltech.edu).

H. Gharibyan is with the Stanford Institute for Theoretical Physics, Stanford University, CA 94305 USA (email: hrant@ stanford.edu).

M. Walter is with the Stanford Institute for Theoretical Physics, Stanford University, CA 94305 USA (email: michael.walter@stanford.edu).

\footnotetext{
${ }^{1}$ In fact, not even post-classical assistance like entanglement or general non-signaling correlations increase the capacity [2], [3].
}

$\left\{\mathcal{J}_{X \rightarrow Y}^{i}\right\}_{i \in I}$ with index set $I$, the asymptotic IID capacity was determined to [5], [6],

$$
C\left(\Lambda_{X \rightarrow Y}\right)=\sup _{P_{X}} \inf _{i \in I} I(X: Y)_{\mathcal{J}^{i}(P)} .
$$

Furthermore, and in contrast to the case of a single channel, feedback from the receiver to the sender improves this to [7], [8],

$$
C_{F}\left(\Lambda_{X \rightarrow Y}\right)=\inf _{i \in I} C\left(\mathcal{J}_{X \rightarrow Y}^{i}\right) .
$$

Now, unlike in the classical case, IID quantum channels have various different asymptotic capacities and only in special cases formulas to compute these are known [9], [2], [10], [11], [12], [13]. For the entanglement-assisted quantum capacity, however, a formula similar to (1) is available and for that reason it is considered to be the natural quantum analogue of the capacity of classical channels. For a fixed quantum channel $\mathcal{N}_{A \rightarrow B}$, the entanglement-assisted quantum capacity is given by the quantum mutual information [2],

$$
Q_{E}\left(\mathcal{N}_{A \rightarrow B}\right)=\sup _{\rho} \frac{1}{2} I\left(A^{\prime}: B\right)_{\mathcal{N}(\rho)},
$$

optimized over all purified input distributions $\rho_{A^{\prime} A}$. Like in the classical case, feedback assistance from the receiver to the sender does not increase this capacity [14]. ${ }^{2}$ By quantum teleportation [16] and superdense coding [17], the corresponding entanglement-assisted classical capacity is the same up to a factor of two.

In this paper we study the entanglement-assisted capacities of compound quantum channels $\Pi_{A \rightarrow B}=\left\{\mathcal{N}_{A \rightarrow B}^{i}\right\}_{i \in I}$, where each $\mathcal{N}_{A \rightarrow B}^{i}$ is a quantum channel between finitedimensional input and output spaces $A$ and $B$, respectively, and where $I$ is an arbitrary index set. In full analogy to the classical case (2)-(3), we show that the compound quantum capacity without feedback is given by

$$
Q_{E}\left(\Pi_{A \rightarrow B}\right)=\sup _{\rho} \inf _{i \in I} \frac{1}{2} I\left(A^{\prime}: B\right)_{\mathcal{N}^{i}(\rho)},
$$

and that feedback improves the capacity to

$$
Q_{E, F}\left(\Pi_{A \rightarrow B}\right)=\inf _{i \in I} Q_{E}\left(\mathcal{N}_{A \rightarrow B}^{i}\right) .
$$

Here, the first formula holds for general compounds whereas we can only show the second formula for finite compound channels, $|I|<\infty .{ }^{3}$ In the process of deriving (5)-(6) we

\footnotetext{
${ }^{2}$ As shown in [15], the quantum capacity assisted by non-signaling correlations is also given by (4).

${ }^{3}$ The feedback capacity of general classical compound channels has only been determined recently [8].
} 
also determine the entanglement-assisted compound capacities when either the sender or the receiver is informed about the channel used for communication (this is again in full analogy to the classical case):

$$
\begin{aligned}
& Q_{E, I R}\left(\Pi_{A \rightarrow B}\right)=Q_{E}\left(\Pi_{A \rightarrow B}\right) \text { as well as } \\
& Q_{E, I S}\left(\Pi_{A \rightarrow B}\right)=Q_{E, F}\left(\Pi_{A \rightarrow B}\right) .
\end{aligned}
$$

Prior work: The plain classical and quantum capacities of compound quantum channels were studied in [18], [19], [20] and [21], [22], [23], respectively. In [24], [25] the plain and entanglement-assisted classical capacities of a class of quantum channels with long-term memory were determined. As we will see the latter result is equivalent to formula (5) for finite compounds.

Techniques: For our proofs we follow the decoupling approach to quantum information theory [26], [27], [28], [29], [30]. In particular we make use of one-shot decoupling bounds [31] in terms of smooth entropies [32], [33]. We also utilize various techniques developed in aforementioned references about compound quantum channels.

\section{OVERVIEW OF RESUlTS}

Here we introduce the setup more formally and give a summary of our results and methods. Let $\Pi_{A \rightarrow B}=\left\{\mathcal{N}_{A \rightarrow B}^{i}\right\}_{i \in I}$ be a finite-dimensional compound quantum channel (i.e., a collection of quantum channels with fixed and finite-dimensional input and output systems, labeled by some index set $I$ ). Our goal is the quantification of the information-theoretic power of such channels. In particular, we determine their asymptotic IID capacities when free entanglement-assistance between the sender and the receiver in the form of maximally entangled states $\Phi_{A_{1} B_{1}}^{+}$is available.

a) Uninformed users: An entanglement transmission code for $\Pi_{A \rightarrow B}$ is given by a quadruple $\left\{M_{0}, M_{1}, \mathcal{E}, \mathcal{D}\right\}$, where $M_{0}$ is the local dimension of a maximally entangled state $\Phi_{A_{0} R}^{+}$that is to be transmitted, $M_{1}$ denotes the local dimension of the entanglement-assistance $\Phi_{A_{1} B_{1}}^{+}$, and the quantum channels $\mathcal{E}, \mathcal{D}$ are the encoding and decoding operations, respectively. We say that a triple $(R, n, \delta)$ is achievable for $\Pi_{A \rightarrow B}$ if there exists an entanglement transmission code with

$$
\begin{aligned}
\frac{1}{n} \log M_{0} & \geq R \text { and } \inf _{i \in I} F\left(\Phi_{A_{0} R}^{+}, \mathcal{D}_{B B_{1} \rightarrow A_{0}} \circ\right. \\
\left.\mathcal{N}_{A \rightarrow B}^{i} \circ \mathcal{E}_{A_{0} A_{1} \rightarrow A}\left(\Phi_{A_{0} R}^{+} \otimes \Phi_{A_{1} B_{1}}^{+}\right)\right) & \geq 1-\delta .
\end{aligned}
$$

Here, $R$ is the rate of the code, $n \in \mathbb{N}$ the number of channel uses, and $\delta>0$ the tolerated error measured in terms of Uhlmann's fidelity $F(\rho, \sigma):=\|\sqrt{\rho} \sqrt{\sigma}\|_{1}^{2}$. This means that for every channel $\mathcal{N}_{A \rightarrow B}^{i}$ in the compound, the fidelity of transmission should be high, averaged over a uniform message of size $M_{0}$. In the asymptotic IID limit the entanglementassisted capacity of the compound quantum channel $\Pi_{A \rightarrow B}$ is then defined as

$$
\begin{aligned}
& Q_{E}\left(\Pi_{A \rightarrow B}\right):=\lim _{\delta \rightarrow 0} \liminf _{n \rightarrow \infty} \\
& \quad \sup \left\{R:(R, n, \delta) \text { is achievable for } \Pi_{A \rightarrow B}\right\} .
\end{aligned}
$$

Slightly different definitions of entanglement transmission codes (7) and, correspondingly, channel capacities (8) are possible as well and we point to [34] for an in-depth discussion. We prove the following formula for $Q_{E}\left(\Pi_{A \rightarrow B}\right)$ :

Theorem 1. Let $\Pi_{A \rightarrow B}=\left\{\mathcal{N}_{A \rightarrow B}^{i}\right\}_{i \in I}$ be a compound channel with arbitrary index set I. Then, we have

$$
Q_{E}\left(\Pi_{A \rightarrow B}\right)=\frac{1}{2} \sup _{\rho} \inf _{i \in I} I\left(A^{\prime}: B\right)_{\mathcal{N}^{i}(\rho)},
$$

where the supremum is over all purified input distributions $\rho_{A^{\prime} A}$ with $A \cong A^{\prime}$.

To prove the converse, we rely on the quantum generalization of the meta converse for channel coding from [3], together with some extensions which were inspired by the work [25]. To prove achievability, our starting point is a oneshot coding theorem for fixed channels [30] that is formulated in terms of smooth entropies. These entropies were introduced to study structureless (non-IID) resources [32] and have many properties similar to the von Neumann entropy [33]. By applying the one-shot result to the average channel $\bar{\Pi}_{A \rightarrow B}:=\frac{1}{\mid I} \sum_{i \in I} \mathcal{N}_{A \rightarrow B}^{i}$ associated with a finite compound channel (cf. the work [21]) and exploiting basic properties of smooth entropies, we obtain a one-shot coding theorem for finite compound channels. In the asymptotic limit, this establishes Theorem 1 for finite compound channels. In light of this proof and the equivalence between the entanglement-assisted transmission of classical and quantum information, as discussed below, this result can be seen to be equivalent to the work [25] where the entanglement assisted classical capacity of a class of quantum channels of the form $\hat{\mathcal{N}}_{A^{n} \rightarrow B^{n}}=\sum_{i \in I} p_{i}\left(\mathcal{N}_{A \rightarrow B}^{i}\right)^{\otimes n}$ with $\left\{p_{i}\right\}_{i \in I}$ a finite probability distribution was determined (using different techniques). To extend the proof to arbitrary compound channels we use a discretization argument via net techniques (similarly to what is done in, e.g., [23]). The basic reason why this works is that the random coding schemes that we make use of have a well behaved error dependence, together with the mutual information being nicely continuous.

b) Informed receiver: When the receiver but not the sender knows which one of the channels $\mathcal{N}_{A \rightarrow B}^{i}$ from the compound will be used for the information transmission, the decoder $\mathcal{D}_{B B_{1} \rightarrow A_{0}}^{i}$ can depend on the channel and thus (7) becomes

$$
\begin{gathered}
\frac{1}{n} \log M_{0} \geq R \text { and } \inf _{i \in I} F\left(\Phi_{A_{0} R}^{+}, \mathcal{D}_{B B_{1} \rightarrow A_{0}}^{i} \circ\right. \\
\left.\mathcal{N}_{A \rightarrow B}^{i} \circ \mathcal{E}_{A_{0} A_{1} \rightarrow A}\left(\Phi_{A_{0} R}^{+} \otimes \Phi_{A_{1} B_{1}}^{+}\right)\right) \geq 1-\delta,
\end{gathered}
$$

The corresponding capacity $Q_{E, I R}$ is then defined as in (8) and we find that it does not increase compared to the uninformed case.

Theorem 2. Let $\Pi_{A \rightarrow B}=\left\{\mathcal{N}_{A \rightarrow B}^{i}\right\}_{i \in I}$ be a compound channel with arbitrary index set $I$. Then, we have

$$
Q_{E, I R}\left(\Pi_{A \rightarrow B}\right)=Q_{E}\left(\Pi_{A \rightarrow B}\right) .
$$

For the proof we simply note that the converse established for the uninformed case does not rely on the decoder being uninformed and thus holds verbatim for informed receivers. 
c) Informed sender: When the sender but not the receiver is aware of which channel $\mathcal{N}_{A \rightarrow B}^{i}$ from the compound will be used for the information transmission, the encoder $\mathcal{E}_{A_{0} A_{1} \rightarrow A}^{i}$ may now depend on $i \in I$ and thus (7) becomes

$$
\begin{aligned}
& \frac{1}{n} \log M_{0} \geq R \text { and } \inf _{i \in I} F\left(\Phi_{A_{0} R}^{+}, \mathcal{D}_{B B_{1} \rightarrow A_{0}} \circ\right. \\
&\left.\mathcal{N}_{A \rightarrow B}^{i} \circ \mathcal{E}_{A_{0} A_{1} \rightarrow A}^{i}\left(\Phi_{A_{0} R}^{+} \otimes \Phi_{A_{1} B_{1}}^{+}\right)\right) \geq 1-\delta .
\end{aligned}
$$

The corresponding capacity $Q_{E, I S}$ is then defined as in (8) and we find that in general the capacity increases. More precisely, we show that infimum and supremum in the formula (9) can be interchanged, so that the entanglement-assisted quantum capacity of a compound equals the infimum of the capacities of its constituents:

Theorem 3. Let $\Pi_{A \rightarrow B}=\left\{\mathcal{N}_{A \rightarrow B}^{i}\right\}_{i \in I}$ be a compound channel with arbitrary index set $I$. Then, we have

$$
Q_{E, I S}\left(\Pi_{A \rightarrow B}\right)=\inf _{i \in I} Q_{E}\left(\mathcal{N}_{A \rightarrow B}^{i}\right)
$$

where

$$
Q_{E}\left(\mathcal{N}_{A \rightarrow B}^{i}\right)=\frac{1}{2} \max _{\rho} I\left(A^{\prime}: B\right)_{\mathcal{N}^{i}(\rho)}
$$

is the entanglement-assisted capacity of the $i$-th channel as determined in [2].

The converse direction follows directly from the converse for a fixed channel [2], since the capacity can not be higher than the capacity of any single channel in the compound. For the achievability, we derive new one-shot decoupling bounds that then imply the existence of a universal decoder by a standard argument via Uhlmann's theorem. As before we reduce from general to finite compounds by using a discretization argument via net techniques.

d) Feedback: We also solve the scenario with free feedback from receiver to sender. ${ }^{4}$ The first round of a feedbackassisted entanglement transmission code starts with a quantum or classical feedback message $X_{A}^{(0)}$ that is correlated with some $X_{B}^{(0)}$ at the receiver, followed by an encoder $\mathcal{E}_{X_{A}^{(0)} A_{0} A_{1} \rightarrow A}$, the channel $\mathcal{N}_{A \rightarrow B}^{i}$ from the compound, and a decoder $\mathcal{D}_{X_{B}^{(0)} B B_{1} \rightarrow A_{0}}$. Now, for $n$ channel uses the procedure repeats $n$-times. The corresponding capacity $Q_{E, F}$ is then defined as in (8), where we have an application of $n$-rounds of encoders and decoders and the fidelity bound in (7) holds for these $n$-rounds [14]. We find that the corresponding feedback capacity is the same as in the case of informed senders (at least for finite compounds). Therefore, in analogy to the classical case, feedback in general increases the entanglement-assisted quantum capacity of compound channels.

Theorem 4. Let $\Pi_{A \rightarrow B}=\left\{\mathcal{N}_{A \rightarrow B}^{i}\right\}_{i \in I}$ be a compound channel with finite index set $|I|<\infty$. Then, we have

$$
Q_{E, F}\left(\Pi_{A \rightarrow B}\right)=Q_{E, I S}\left(\Pi_{A \rightarrow B}\right) .
$$

The converse direction again follows from the converse of the corresponding result for a single channel [14]. For the

\footnotetext{
${ }^{4}$ Since we assume free entanglement-assistance, classical and quantum feedback are equivalent by quantum teleportation [16] and superdense coding [17].
}

achievability we make use of the channel estimation techniques from [24]. In particular, we use the first $\sqrt{n}$ instances of the channel to estimate the channel on the receiver's side and then use feedback to transfer the channel index $i \in I$ to the sender. This allows to use the informed sender protocol for the remaining $n-\sqrt{n}$ channel uses and leads to the same capacity as in the informed sender case. Unfortunately, it is unclear how to apply this technique for general compounds since in this case the trade-off between the discretization and the channel estimation parameters might not scale well enough. We note that even for classical compound channels this issue has only been resolved relatively recently [8], and we leave it as an open problem in the quantum case.

e) Application: We consider a situation where some channel $\mathcal{N}_{A \rightarrow B}$ used for information transmission has only been characterized up to some precision $\varepsilon>0 .^{5}$ This corresponds to uninformed users (Theorem 1) and by the continuity of the conditional entropy [35], [36] we find that we can still transmit quantum information at a rate of at least

$$
Q_{E}\left(\mathcal{N}_{A \rightarrow B}\right)-\varepsilon \log d_{A}+\left(1+\frac{\varepsilon}{2}\right) h\left(\frac{\varepsilon}{2+\varepsilon}\right)
$$

where $d_{A}$ denotes the input dimension of the quantum channel, and $h(p)$ denotes the binary entropy function. Hence, we can conclude that not knowing the quantum channel perfectly only sightly impacts our ability to transmit quantum information. (This does not just follow from the continuity of the entanglement-assisted channel capacity since we need a universal coding scheme that works for all channels in the $\varepsilon$-neighborhood.)

f) Classical communication: Lastly, we discuss the entanglement-assisted transmission of classical information through the compound quantum channel $\Pi_{A \rightarrow B}$. A classical code for $\Pi_{A \rightarrow B}$ is given by a quadruple $\left\{M_{0}, M_{1},\left\{\mathcal{E}^{m}\right\},\left\{\Lambda^{m}\right\}\right\}$, where $M_{0}$ is the number of classical messages to be transmitted, $M_{1}$ denotes the local dimension of the entanglement-assistance $\Phi_{A_{1} B_{1}}^{+},\left\{\mathcal{E}_{A_{1} \rightarrow A}^{m}\right\}_{m=1}^{M_{0}}$ are the encoding operations for the classical messages, and $\left\{\Lambda_{B B_{1}}^{m}\right\}_{m=1}^{M_{0}}$ the POVM elements of the decoding measurement. Then, for example for the uninformed users setting like in (7), we say that a triple $(R, n, \delta)$ is achievable for $\Pi_{A \rightarrow B}$ if there exists a classical code with

$$
\begin{aligned}
\frac{1}{n} \log M_{0} \geq R \text { and } \inf _{i \in I} \frac{1}{M_{0}} \sum_{m=1}^{M_{0}} \operatorname{tr}\left[\Lambda_{B B_{1}}^{m}\right. \\
\left.\left(\mathcal{N}_{A \rightarrow B}^{i} \circ \mathcal{E}_{A_{1} \rightarrow A}^{m}\left(\Phi_{A_{1} B_{1}}^{+}\right)\right)\right] \geq 1-\delta .
\end{aligned}
$$

This means that for every possible choice $\mathcal{N}_{A \rightarrow B}^{i}$ in the compound, the probability of success to retrieve the message should be high, averaged uniformly over all messages $m \in M_{0}$. The corresponding entanglement-assisted classical capacity $C_{E}$ is then defined in the exact same way as in (8). Now, in the presence of free entanglement-assistance we have quantum teleportation [16] and superdense coding [17] available: this

\footnotetext{
${ }^{5}$ More precisely, we assume that for some fixed input system we know the channel $\mathcal{N}_{A \rightarrow B}$ up to $\varepsilon>0$ in the diamond norm (see Section III for its definition).
} 
allows to transform entanglement transmission codes into classical codes and vice versa. More precisely, following [15, (46) \& Appendix B] we get with superdense coding that,

$\left\{M_{0}, M_{1}, \mathcal{E}, \mathcal{D}\right\}$ with fidelity $1-\delta \Rightarrow$ $\left\{M_{0}^{2}, M_{1} M_{0},\left\{\mathcal{E}^{m}\right\},\left\{\Lambda^{m}\right\}\right\}$ with success probability $1-\delta$,

and vice versa with quantum teleportation that,

$\left\{M_{0}, M_{1},\left\{\mathcal{E}^{m}\right\},\left\{\Lambda^{m}\right\}\right\}$ with success probability $1-\delta \Rightarrow$ $\left\{\sqrt{M_{0}}, M_{1} M_{0}, \mathcal{E}, \mathcal{D}\right\}$ with fidelity $1-\delta$.

Asymptotically this leads to the following channel capacity identities:

$$
2=\frac{C_{E}}{Q_{E}}=\frac{C_{E, I R}}{Q_{E, I R}}=\frac{C_{E, I S}}{Q_{E, I S}}=\frac{C_{E, F}}{Q_{E, F}} .
$$

g) Extensions: Our proofs bring up new tools that can deployed for simplifying previous works about capacities of compound quantum channels [18], [19], [20], [24], [21], [22], [23]. In Section A we present such an argument for the plain quantum capacity of compound quantum channels.

h) Related work: After completion of our work, we have learned about the concurrent work [37], [38] that also determines the entanglement-assisted quantum capacity of compound channels for uninformed users (amongst various other results). However, the key techniques used for the proofs differ significantly. The authors of [37], [38] use Weyl unitaries as special encoding channels to convert the problem of entanglement-assisted compound channels to a question about classical-quantum compound channels. Then, they deploy established universal codes [18] for classical-quantum channels.

\section{NOTATION}

Here, we introduce our notation and give some standard definitions and lemmas that we will throughout this paper. For more about quantum information theory we point to the excellent textbooks [39], [33].

Let $A, B, C, \ldots$ denote finite-dimensional Hilbert spaces, let $d_{A}$ denote the dimension of $A$, and let $d_{A B}=d_{A} d_{B}$ denote the dimension of $A B=A \otimes B$. We write $\mathcal{P}(A)$ for the set of positive semidefinite operators, $\mathcal{S}(A)$ for the set of normalized quantum states, $\mathcal{S}_{\leq}(A)$ for the set of sub-normalized quantum states (i.e., positive semidefinite operators of trace no larger than one). We use $\tau_{A}=I_{A} / d_{A}$ for the maximally mixed state on $A$ and $\Phi_{A A^{\prime}}^{+}=\frac{1}{d_{A}} \sum_{i, j}|i i\rangle\left\langle\left. j j\right|_{A A^{\prime}}\right.$ for a maximally entangled state, where $A^{\prime} \cong A$.

\section{A. Distance measures}

a) For quantum states: We write $\|X\|_{1}:=\operatorname{tr}\left[\sqrt{X^{\dagger} X}\right]$ for the trace norm. The fidelity is defined for arbitrary positive semidefinite operators $\rho, \sigma \in \mathcal{P}(A)$ by $F(\rho, \sigma):=\|\sqrt{\rho} \sqrt{\sigma}\|_{1}^{2}$. Note that for pure states $\rho$ and $\sigma$ we get $F(\rho, \sigma)=|\langle\rho, \sigma\rangle|^{2}$.
A particular version of Uhlmann's theorem is [30, Theorem 3.1],

$$
\begin{aligned}
F\left(\rho_{A}, \sigma_{A}\right) & =\max _{V_{B \rightarrow C}} F\left(V_{B \rightarrow C} \rho_{A B} V_{B \rightarrow C}^{\dagger}, \sigma_{A C}\right) \\
& =\max _{W_{C \rightarrow B}} F\left(\rho_{A B}, W_{C \rightarrow B} \sigma_{A C} W_{C \rightarrow B}^{\dagger}\right),
\end{aligned}
$$

where $\rho_{A B}$ is an arbitrary purification of $\rho_{A}, \sigma_{A C}$ an arbitrary purification of $\sigma_{A}$, and where the maximizations are over partial isometries. For normalized quantum states $\rho, \sigma \in \mathcal{S}(A)$, fidelity and trace norm are related by the Fuchs-van de Graaf inequalities:

$$
\left(1-\frac{\|\rho-\sigma\|_{1}}{2}\right)^{2} \leq F(\rho, \sigma) \leq 1-\frac{1}{4}\|\rho-\sigma\|_{1}^{2} .
$$

As a consequence, one obtains the following lemma, which is central to the decoupling approach to quantum information theory.

Lemma 5 (cf. [30, Corollary 3.2]). Let $\rho_{A B} \in \mathcal{P}(A B)$ and $\sigma_{A C} \in \mathcal{S}(A B)$ be pure states with $\left\|\rho_{A}-\sigma_{A}\right\|_{1} \leq \delta$. Then, there exist partial isometries $V_{B \rightarrow C}$ and $W_{C \rightarrow B}$ such that

$$
\left\|V_{B \rightarrow C} \rho_{A B} V_{B \rightarrow C}^{\dagger}-\sigma_{A C}\right\|_{1} \leq \delta+2 \sqrt{2 \delta}
$$

as well as

$$
\left\|\rho_{A B}-W_{C \rightarrow B} \sigma_{A C} W_{C \rightarrow B}^{\dagger}\right\|_{1} \leq \delta+2 \sqrt{2 \delta} .
$$

A proof can be found in Section A. We note that $\delta+2 \sqrt{2 \delta} \leq$ $4 \sqrt{\delta}$ if $\delta \leq 1$, which will often be the case.

Fidelity and trace norm can be related for arbitrary positive semidefinite operators $\rho, \sigma \in \mathcal{P}(A)$ [32, Lemmas A.2.4 and A.2.6],

$$
\begin{aligned}
& \left(\frac{\operatorname{tr}[\rho]+\operatorname{tr}[\sigma]}{2}-\frac{\|\rho-\sigma\|_{1}}{2}\right)^{2} \leq F(\rho, \sigma) \\
\leq & \frac{(\operatorname{tr}[\rho]+\operatorname{tr}[\sigma])^{2}}{4}-\frac{1}{4}\|\rho-\sigma\|_{1}^{2},
\end{aligned}
$$

which generalizes (14). In particular, the lower bound implies that

$$
F(\rho, \sigma) \geq 1-2\|\rho-\sigma\|_{1} \quad \text { for } \rho \in \mathcal{P}(A), \sigma \in \mathcal{S}(A) .
$$

We will also need the following continuity bound for the fidelity, which follows from, e.g., [40, Lemma B.9]: for $\rho, \sigma, \sigma^{\prime} \in \mathcal{S}_{\leq}(A)$ we have

$$
\begin{aligned}
\left|F(\rho, \sigma)-F\left(\rho, \sigma^{\prime}\right)\right| & \leq 2\left|\sqrt{F(\rho, \sigma)}-\sqrt{F\left(\rho, \sigma^{\prime}\right)}\right| \\
& \leq 2 \sqrt{\left\|\sigma-\sigma^{\prime}\right\|_{1}} .
\end{aligned}
$$

Lastly, for $\rho, \sigma \in \mathcal{S}_{<}(A)$ we need the purified distance $P(\rho, \sigma):=\sqrt{1-F_{*}(\rho, \sigma)}$, defined in terms of the generalized fidelity $F_{*}(\rho, \sigma):=(\sqrt{F(\rho, \sigma)}+\sqrt{(1-\operatorname{tr}[\rho])(1-\operatorname{tr}[\sigma])})^{2}$. We have

$$
\|\rho-\sigma\|_{1} \leq 2 P(\rho, \sigma) \text { for } \rho, \sigma \in \mathcal{S}_{\leq}(A) .
$$


b) For quantum operations: The diamond norm of a super-operator $\mathcal{N}_{A \rightarrow B}$ is defined as

$$
\|\mathcal{N}\|_{\diamond}:=\max _{\rho_{A A^{\prime}} \in \mathcal{S}\left(A A^{\prime}\right)}\left\|\mathcal{N}_{A \rightarrow B}\left(\rho_{A A^{\prime}}\right)\right\|_{1} \text { with } A^{\prime} \cong A .
$$

From the continuity bounds for the fidelity (16) we find for $\mathcal{T}_{A \rightarrow B}, \mathcal{T}_{A \rightarrow B}^{\prime}$ quantum operations (completely positive and trace-nonincreasing maps) and $\rho, \sigma \in \mathcal{S}_{\leq}(A)$ that,

$$
\begin{aligned}
\left|F(\rho, \mathcal{T}(\sigma))-F\left(\rho, \mathcal{T}^{\prime}(\sigma)\right)\right| & \leq 2 \sqrt{\left\|\left(\mathcal{T}-\mathcal{T}^{\prime}\right)(\sigma)\right\|_{1}} \\
& \leq 2 \sqrt{\left\|\mathcal{T}-\mathcal{T}^{\prime}\right\|_{\diamond}}
\end{aligned}
$$

\section{B. Entropies}

a) Von Neumann entropy: For $\rho_{A B} \in \mathcal{S}(A B)$ the von Neumann entropy ${ }^{6}$ of system $A$ is defined by $H(A)_{\rho}:=$ $-\operatorname{tr}\left[\rho_{A} \log \rho_{A}\right]$. The conditional entropy of $A$ given $B$ is $H(A \mid B)_{\rho}:=H(A B)_{\rho}-H(B)_{\rho}$, and the mutual information between $A$ and $B$ is $I(A: B)_{\rho}:=H(A)_{\rho}+H(B)_{\rho}-H(A B)_{\rho}$. All of these functions are continuous, and we will use the following continuity bounds for $\rho_{A B}, \sigma_{A B} \in \mathcal{S}(A B)$ : For the conditional entropy [35], [36], we have

$$
\begin{aligned}
& \left|H(A \mid B)_{\rho}-H(A \mid B)_{\sigma}\right| \\
\leq & 2 T \log d_{A}+(1+T) h\left(\frac{T}{1+T}\right),
\end{aligned}
$$

while for the mutual information [41],

$$
\begin{aligned}
& \left|I(A: B)_{\rho}-I(A: B)_{\sigma}\right| \\
\leq & 2 T \log \min \left\{d_{A}, d_{B}\right\}+2(1+T) h\left(\frac{T}{1+T}\right) .
\end{aligned}
$$

In both cases, $T:=\frac{1}{2}\left\|\rho_{A B}-\sigma_{A B}\right\|_{1}$, and $h(p)$ denotes the binary entropy function.

b) Smooth entropies: For $\rho_{A B} \in \mathcal{S}_{\leq}(A B)$ the conditional min-entropy of $A$ given $B$ is defined as [32]

$$
\begin{aligned}
& H_{\min }(A \mid B)_{\rho} \\
:= & -\log \min \left\{\operatorname{tr}\left[\sigma_{B}\right]: \sigma_{B} \in \mathcal{P}(B), \rho_{A B} \leq I_{A} \otimes \sigma_{B}\right\} .
\end{aligned}
$$

The $\varepsilon$-smooth conditional min-entropy is defined by the following optimization over nearby states [42]:

$$
\begin{aligned}
& H_{\min }^{\varepsilon}(A \mid B)_{\rho} \\
:= & \sup \left\{H_{\min }(A \mid B)_{\tilde{\rho}}: \widetilde{\rho} \in \mathcal{S}_{\leq}(A B), P(\widetilde{\rho}, \rho) \leq \varepsilon\right\}
\end{aligned}
$$

Note that we have $H_{\min }^{0}(A \mid B)_{\rho}=H_{\min }(A \mid B)_{\rho}$ since the purified distance defines a metric on the sub-normalized states. The (smooth) conditional max-entropy for $\varepsilon \geq 0$ is defined by duality as [43], [42],

$$
H_{\max }^{\varepsilon}(A \mid B)_{\rho}:=-H_{\min }^{\varepsilon}(A \mid C)_{\rho},
$$

where the smooth conditional min-entropy on the right-hand side is evaluated with respect to the reduced density matrix $\rho_{B C}$ of an arbitrary purification $\left|\rho_{A B C}\right\rangle$ of $\rho_{A B}$. It holds that [42]

$$
\begin{aligned}
& H_{\max }^{\varepsilon}(A \mid B)_{\rho} \\
= & \sup \left\{H_{\max }(A \mid B)_{\widetilde{\rho}}: \widetilde{\rho} \in \mathcal{S}_{\leq}(A B), P(\widetilde{\rho}, \rho) \leq \varepsilon\right\} .
\end{aligned}
$$

${ }^{6}$ All logarithms are to base 2 . where $H_{\max }(A \mid B)=H_{\max }^{0}(A \mid B)$. We will make use of the following lemmas about the smooth conditional min- and maxentropy of convex combination quantum states.

Lemma 6. Let $\left\{\rho_{A B}^{i}\right\}_{i=1}^{N} \subseteq \mathcal{S}(A B)$ be quantum states, $\left\{p_{i}\right\}_{i=1}^{N}$ a probability distribution, and $\rho_{A B}=\sum_{i=1}^{N} p_{i} \rho_{A B}^{i}$. Then, we have

$$
H_{\min }^{\varepsilon}(A \mid B)_{\rho} \geq \min _{i} H_{\min }^{\varepsilon}(A \mid B)_{\rho^{i}} .
$$

Lemma 7. Let $\left\{\rho_{A B}^{i}\right\}_{i=1}^{N} \subseteq \mathcal{S}(A B)$ be quantum states, $\left\{p_{i}\right\}_{i=1}^{N}$ a probability distribution, and $\rho_{A B}=\sum_{i=1}^{N} p_{i} \rho_{A B}^{i}$. Then, we have

$$
H_{\max }^{\varepsilon}(A \mid B)_{\rho} \leq \max _{i} H_{\max }^{\varepsilon}(A \mid B)_{\rho^{i}}+2 \log N .
$$

Lemmas 6 and 7 are proved in Section A. The smooth conditional min- and max-entropy satisfy the following asymptotic equipartition property [44], [33]:

$$
\begin{aligned}
& \frac{1}{n} H_{\min }^{\varepsilon}\left(A^{n} \mid B^{n}\right)_{\rho^{\otimes n}} \geq H(A \mid B)_{\rho}-\frac{\delta(\varepsilon, \rho)}{\sqrt{n}}, \\
& \frac{1}{n} H_{\max }^{\varepsilon}\left(A^{n} \mid B^{n}\right)_{\rho^{\otimes n}} \leq H(A \mid B)_{\rho}+\frac{\delta(\varepsilon, \rho)}{\sqrt{n}} .
\end{aligned}
$$

where $\rho_{A B} \in \mathcal{S}(A B)$ and $n \in \mathbb{N}$. For $n \geq \frac{8}{5} \log \frac{2}{\varepsilon^{2}}$ the convergence parameter can be bounded as:

$$
\delta(\varepsilon, \rho) \leq 4 \log \left(2 \sqrt{d_{A}}+1\right) \sqrt{\log \frac{2}{\varepsilon^{2}}} .
$$

For technical reasons we will also work with the conditional collision entropy defined as [32],

$$
H_{2}(A \mid B)_{\rho}:=-\log \min _{\sigma \in \mathcal{S}(B)} \operatorname{tr}\left[\left(\sigma_{B}^{-1 / 4} \rho_{A B} \sigma_{B}^{-1 / 4}\right)^{2}\right],
$$

where $\rho_{A B} \in \mathcal{S}_{\leq}(A B)$. We have $H_{2}(A \mid B)_{\rho} \geq H_{\min }(A \mid B)_{\rho}$.

\section{UNINFORMED USERS AND INFORMED RECEIVER}

Since the two capacities advertised in Theorems 1 and 2 match, it suffices to prove a coding theorem for uninformed users together with a converse bound for the informed receiver scenario. We start in Section IV-A by establishing a oneshot coding theorem for finite compound channels by a wellknown reduction to a single average channel and properties of smooth entropies. In Section IV-B we obtain the direct part of Theorem 1 by using our one-shot result in the limit of many channel uses combined with discretization techniques. Lastly, in Section IV-C we establish the converse part of Theorem 2.

\section{A. One-shot coding theorem}

To establish our one-shot coding result, we use an equivalence between finite compound channels and average channels, whose analogue for the plain quantum capacity has been observed previously in [21, Lemma II.1]. ${ }^{7}$ Let $\Pi_{A \rightarrow B}=$ $\left\{\mathcal{N}_{A \rightarrow B}^{i}\right\}_{i=1}^{N}$ be a finite compound channel and consider the average channel $\bar{\Pi}_{A \rightarrow B}:=\frac{1}{N} \sum_{i=1}^{N} \mathcal{N}_{A \rightarrow B}^{i}$. If $\mathcal{E}$ and

${ }^{7}$ We must keep in mind that this equivalence is only known to hold true for weak capacities as defined in (8), and not for strong converse capacities. 
$\mathcal{D}$ denote encoder and decoder for $\bar{\Pi}_{A \rightarrow B}$ that achieve an entanglement fidelity of

$$
\begin{aligned}
& F\left(\Phi_{A_{0} R}^{+}, \mathcal{D} \circ \bar{\Pi} \circ \mathcal{E}\left(\Phi_{A_{0} R}^{+} \otimes \Phi_{A_{1} B_{1}}^{+}\right)\right) \\
& =\frac{1}{N} \sum_{i=1}^{N} F\left(\Phi_{A_{0} R}^{+}, \mathcal{D} \circ \mathcal{N}^{i} \circ \mathcal{E}\left(\Phi_{A_{0} R}^{+} \otimes \Phi_{A_{1} B_{1}}^{+}\right)\right) \\
& \geq 1-\delta
\end{aligned}
$$

then it is immediate that, for all $i=1, \ldots, N$,

$$
F\left(\Phi_{A_{0} R}^{+}, \mathcal{D} \circ \mathcal{N}^{i} \circ \mathcal{E}\left(\Phi_{A_{0} R}^{+} \otimes \Phi_{A_{1} B_{1}}^{+}\right)\right) \geq 1-N \delta .
$$

Thus, the entanglement infidelity does increase by no more than a constant factor $N$ when we apply a code to the compound channel instead of the average channel. Conversely, any code for the compound channel achieves the same entanglement fidelity for the average channel.

We now prove the following one-shot coding theorem for finite compound channels:

Theorem 8. For any finite compound channel $\Pi_{A \rightarrow B}=$ $\left\{\mathcal{N}_{A \rightarrow B}^{i}\right\}_{i=1}^{N}$, pure state $\rho_{A A^{\prime}}$, integers $M_{0}$ and $M_{1}$, and $\varepsilon>0$, there exist quantum operations $\mathcal{E}_{A_{0} A_{1} \rightarrow A}$ and $\mathcal{D}_{B B_{1} \rightarrow A_{0}}$, where $d_{A_{0}}=M_{0}$ and $d_{A_{1}}=d_{B_{1}}=M_{1}$, such that

$$
\begin{aligned}
& \min _{i} F\left(\Phi_{A_{0} R}^{+}, \mathcal{D} \circ \mathcal{N}^{i} \circ \mathcal{E}\left(\Phi_{A_{0} R}^{+} \otimes \Phi_{A_{1} B_{1}}^{+}\right)\right) \\
\geq & 1-4 N \sqrt{2 \sqrt{\delta_{1}}+\delta_{2}},
\end{aligned}
$$

where

$$
\begin{aligned}
\delta_{1}= & 3 \cdot 2^{-\frac{1}{2}\left(H_{\min }^{\varepsilon}(A)_{\rho}-\log M_{0}-\log M_{1}\right)}+24 \varepsilon, \\
\delta_{2}= & 3 \cdot 2^{-\frac{1}{2}\left(-\max _{i} H_{\max }^{\varepsilon}\left(A^{\prime} \mid B\right)_{\mathcal{N}^{i}(\rho)}-2 \log N-\log M_{0}+\log M_{1}\right)} \\
& +24 \varepsilon .
\end{aligned}
$$

Here, $H_{\min }^{\varepsilon}(A)_{\rho}$ stands for the smooth min-entropy, defined as in (22) with $B$ a one-dimensional system.

Proof. We apply the one-shot coding theorem [30, Theorem 3.14 , cf. Theorem 3.15] to the average channel $\bar{\Pi}_{A \rightarrow B}$ to obtain quantum operations $\mathcal{E}$ and $\mathcal{D}$ such that

$$
\left\|\mathcal{D} \circ \bar{\Pi} \circ \mathcal{E}\left(\Phi_{A_{0} R}^{+} \otimes \Phi_{A_{1} B_{1}}^{+}\right)-\Phi_{A_{0} R}^{+}\right\|_{1} \leq 2 \sqrt{2 \sqrt{\delta_{1}}+\delta_{2}}
$$

where

$$
\begin{aligned}
& \delta_{1}=3 \cdot 2^{-\frac{1}{2}\left(H_{\min }^{\varepsilon}(A)_{\rho}-\log M_{0}-\log M_{1}\right)}+24 \varepsilon, \\
& \delta_{2}=3 \cdot 2^{-\frac{1}{2}\left(-H_{\max }^{\varepsilon}\left(A^{\prime} \mid B\right)_{\bar{\Pi}(\rho)}-\log M_{0}+\log M_{1}\right)}+24 \varepsilon .
\end{aligned}
$$

Using the relation between the fidelity and the trace distance in (15), we obtain

$$
F\left(\Phi_{A_{0} R}^{+}, \mathcal{D} \circ \bar{\Pi} \circ \mathcal{E}\left(\Phi_{A_{0} R}^{+} \otimes \Phi_{A_{1} B_{1}}^{+}\right)\right) \geq 1-4 \sqrt{2 \sqrt{\delta_{1}}+\delta_{2}} .
$$

and thus, from (29),

$$
\begin{aligned}
& F\left(\Phi_{A_{0} R}^{+}, \mathcal{D} \circ N^{i} \circ \mathcal{E}\left(\Phi_{A_{0} R}^{+} \otimes \Phi_{A_{1} B_{1}}^{+}\right)\right) \\
& \geq 1-4 N \sqrt{2 \sqrt{\delta_{1}}+\delta_{2}}
\end{aligned}
$$

for all $i=1, \ldots, N$. On the other hand, we have that $\bar{\Pi}(\rho)=$ $\frac{1}{N} \sum_{i=1}^{N} \mathcal{N}_{i}(\rho)$. Thus, we can apply Lemma 7 in Section A, which asserts that

$$
H_{\max }^{\varepsilon}\left(A^{\prime} \mid B\right)_{\bar{\Pi}(\rho)} \leq \max _{i} H_{\max }^{\varepsilon}\left(A^{\prime} \mid B\right)_{\mathcal{N}_{i}(\rho)}+2 \log N
$$

and conclude that

$$
\begin{aligned}
\delta_{2} \leq & 3 \cdot 2^{-\frac{1}{2}\left(-\max _{i} H_{\max }^{\varepsilon}\left(A^{\prime} \mid B\right)_{\mathcal{N}_{i}(\rho)}-2 \log N-\log M_{0}+\log M_{1}\right)} \\
& +24 \varepsilon .
\end{aligned}
$$

\section{B. Achievability}

We now establish the direct part of Theorem 1:

Lemma 9. Let $\Pi_{A \rightarrow B}=\left\{\mathcal{N}_{A \rightarrow B}^{i}\right\}_{i \in I}$ be a compound channel with arbitrary index set $I$. Then, we have

$$
Q_{E}\left(\Pi_{A \rightarrow B}\right) \geq \frac{1}{2} \sup _{\rho} \inf _{i \in I} I\left(A^{\prime}: B\right)_{\mathcal{N}^{i}(\rho)},
$$

where the supremum is over all purified input distributions $\rho_{A^{\prime} A}$ with $A \cong A^{\prime}$.

Proof. Let $\rho_{A A^{\prime}}$ be a pure state, $\Delta>0$, and

$$
R=\frac{1}{2} \inf _{i \in I} I\left(A^{\prime}: B\right)_{\mathcal{N}^{i}(\rho)}-\Delta .
$$

We will show that for any $\delta>0$ there exists $n_{0}$ such that, for all $n \geq n_{0}$, the triple $(R, n, \delta)$ is achievable for $\Pi_{A \rightarrow B}$. If $R \leq 0$ then there is nothing to show, thus we may assume that $\bar{R}>0$.

To reduce to finite compound channels, we use the discretization result [21, Lemma V.2], which asserts that for any $\nu>0$ there exists a finite compound channel $\widetilde{\Pi}_{A \rightarrow B}=$ $\left\{\widetilde{\mathcal{N}}_{A \rightarrow B}^{j}\right\}_{j=1}^{N}$ of cardinality $N \leq(6 / \nu)^{2 d_{A B}^{2}}$ with the property that for any $\mathcal{N}^{i} \in \Pi$ there exists some $\widetilde{\mathcal{N}}^{j} \in \widetilde{\Pi}$ such that $\left\|\left(\mathcal{N}^{i}\right)^{\otimes k}-\left(\tilde{\mathcal{N}}^{j}\right)^{\otimes k}\right\|_{\diamond} \leq k \nu$ for all $k$, and vice versa (that is, for any $\widetilde{\mathcal{N}}^{j} \in \widetilde{\Pi}$ there exists some $\mathcal{N}^{i} \in \Pi$ such that $\left\|\left(\mathcal{N}^{i}\right)^{\otimes k}-\left(\widetilde{\mathcal{N}}^{j}\right)^{\otimes k}\right\|_{\diamond} \leq k \nu$ for all $\left.k\right)$. We shall choose $\nu=1 / n^{2}$. Then, the discretization has cardinality $N \leq(3 n)^{4 d_{A B}^{2}}$. Moreover, $\left\|\mathcal{N}^{i}-\tilde{\mathcal{N}}^{j}\right\|_{\diamond} \leq 1 / n^{2}$ for any pair of channels as above, and thus (20) implies that

$$
\begin{aligned}
& \left|\inf _{i \in I} I\left(A^{\prime}: B\right)_{\mathcal{N}^{i}(\rho)}-\min _{j=1, \ldots, N} I\left(A^{\prime}: B\right)_{\widetilde{\mathcal{N}}^{j}(\rho)}\right| \\
\leq & \frac{1}{n^{2}} \log d_{A}+\left(1+\frac{1}{2 n^{2}}\right) h\left(\frac{1}{2 n^{2}+1}\right) .
\end{aligned}
$$

As a consequence,

$$
R \leq \frac{1}{2} \min _{j=1, \ldots, N} I\left(A^{\prime}: B\right)_{\widetilde{\mathcal{N}}^{j}(\rho)}-\frac{\Delta}{2}
$$

for $n$ sufficiently large (depending only on $d_{A}$ and $\Delta$ ). Let us assume that this is the case.

We now apply our one-shot coding result, Theorem 8, to $\widetilde{\Pi}_{A \rightarrow B}^{\otimes n}=\left\{\left(\widetilde{\mathcal{N}}_{A \rightarrow B}^{j}\right)^{\otimes n}\right\}_{j=1}^{N}, \rho_{A A^{\prime}}^{\otimes n}, M_{0}=\left\lceil 2^{n R}\right\rceil, M_{1}=$ $\left\lceil 2^{n\left(H(A)_{\rho}-R-\Delta / 2\right)}\right\rceil$. For all $\varepsilon>0$, we obtain an encoder $\mathcal{E}_{A_{0} A_{1} \rightarrow A^{n}}$ and a decoder $\mathcal{D}_{B^{n} B_{1} \rightarrow A_{0}}$ such that

$$
\begin{aligned}
& \min _{j=1, \ldots, N} F\left(\Phi_{A_{0} R}^{+}, \mathcal{D} \circ\left(\tilde{\mathcal{N}}^{j}\right)^{\otimes n} \circ \mathcal{E}\left(\Phi_{A_{0} R}^{+} \otimes \Phi_{A_{1} B_{1}}^{+}\right)\right) \\
& \geq 1-4 N \sqrt{2 \sqrt{\delta_{1}}+\delta_{2}},
\end{aligned}
$$

where

$$
\begin{aligned}
& \delta_{1}=3 \cdot 2^{-\frac{1}{2}\left(H_{\min }^{\varepsilon}\left(A^{n}\right)_{\rho \otimes n}-\log M_{0}-\log M_{1}\right)}+24 \varepsilon, \\
& \delta_{2}=3 \cdot 2^{-\frac{1}{2}\left(-\max _{j} H_{\max }^{\varepsilon}\left(A^{\prime n} \mid B^{n}\right)_{\left(\widetilde{\mathcal{N}}^{j}(\rho)\right) \otimes n}-2 \log N\right)} \\
& \cdot 2^{-\frac{1}{2}\left(-\log M_{0}+\log M_{1}\right)}+24 \varepsilon \text {. }
\end{aligned}
$$


We now choose ${ }^{8} \varepsilon=1 /(n N)^{4}$. Since $\varepsilon$ decays only polynomially with $n$, the asymptotic equipartition property (25) together with (27) and the estimate (30) implies that

$$
\delta_{1} \leq 3 \cdot 2^{-\frac{n}{2}\left(\frac{\Delta}{2}-\frac{2}{n}-\frac{\delta(\varepsilon, \rho)}{\sqrt{n}}\right)}+24 \varepsilon \leq 3 \cdot 2^{-n \frac{\Delta}{8}}+24 \varepsilon \leq 25 \varepsilon
$$

for sufficiently large $n$ (depending only $d_{A B}, \Delta$ and $R$ ). Likewise, using (26) instead of (25) we obtain that

$$
\begin{aligned}
\delta_{2} & \leq 3 N \cdot 2^{-\frac{n}{2}\left(\frac{\Delta}{2}-\frac{1}{n}-\frac{\delta\left(\varepsilon, \overline{\mathcal{N}}^{j}(\rho)\right)}{\sqrt{n}}\right)}+24 \varepsilon \\
& \leq 3 N \cdot 2^{-n \frac{\Delta}{8}}+24 \varepsilon \\
& \leq 25 \varepsilon,
\end{aligned}
$$

where we use that $N$ grows only polynomially with $n$. By inserting the two bounds into (31), we obtain that

$$
\begin{aligned}
& F\left(\Phi_{A_{0} R}^{+}, \mathcal{D} \circ\left(\tilde{\mathcal{N}}^{j}\right)^{\otimes n} \circ \mathcal{E}\left(\Phi_{A_{0} R}^{+} \otimes \Phi_{A_{1} B_{1}}^{+}\right)\right) \\
\geq & 1-4 N \sqrt{2 \sqrt{25 \varepsilon}+25 \varepsilon} \geq 1-24 N \varepsilon^{1 / 4} \\
= & 1-\frac{24}{n} .
\end{aligned}
$$

At last, we relate this to the entanglement fidelity for the original compound channel. Using (18) and recalling that $\nu=1 / n^{2}$, we find that

$$
\begin{aligned}
& \inf _{i \in I} F\left(\Phi_{A_{0} R}^{+}, \mathcal{D} \circ\left(\mathcal{N}^{i}\right)^{\otimes n} \circ \mathcal{E}\left(\Phi_{A_{0} R}^{+} \otimes \Phi_{A_{1} B_{1}}^{+}\right)\right) \\
\geq & 1-\frac{24}{n}-\frac{2}{\sqrt{n}}
\end{aligned}
$$

since for any $\mathcal{N}^{i} \in \Pi$ there exist $\widetilde{\mathcal{N}}^{j} \in \widetilde{\Pi}$ such that $\|\left(\mathcal{N}^{i}\right)^{\otimes n}-$ $\left(\widetilde{\mathcal{N}}^{j}\right)^{\otimes n} \|_{\diamond} \leq n \nu=1 / n$. We conclude that, for any $\delta>0$ and sufficiently large $n,(R, \delta, n)$ is a valid triple for the compound channel $\Pi_{A \rightarrow B}$.

\section{Converse}

The following lemma establishes the converse direction of Theorem 2. The proof uses ideas from [45], [3], [25].

Lemma 10. Let $\Pi_{A \rightarrow B}=\left\{\mathcal{N}_{A \rightarrow B}^{i}\right\}_{i \in I}$ be a compound channel with arbitrary index set $I$. Then, we have

$$
Q_{E, I R}\left(\Pi_{A \rightarrow B}\right) \leq \frac{1}{2} \sup _{\rho} \inf _{i \in I} I\left(A^{\prime}: B\right)_{\mathcal{N}^{i}(\rho)}
$$

where the supremum is over all purified input distributions $\rho_{A^{\prime} A}$ with $A \cong A^{\prime}$.

Proof. In view of (12), it suffices to argue that

$$
C_{E, I R}\left(\Pi_{A \rightarrow B}\right) \leq \sup _{\rho} \inf _{i \in I} I\left(A^{\prime}: B\right)_{\mathcal{N}^{i}(\rho)},
$$

where $C_{E, I R}$ stands for the entanglement-assisted classical capacity with informed receiver as discussed in Section II. Thus, let $(R, n, \delta)$ be an achievable triple for entanglementassisted classical communication in the informed receiver scenario, with corresponding code $\left(M_{0}, M_{1},\left\{\mathcal{E}^{m}\right\},\left\{\Lambda^{m, i}\right\}_{m, i}\right)$, where $\left\{\Lambda^{m, i}\right\}_{m=1}^{M_{0}}$ is a POVM for each fixed $i \in I$. Let $\rho_{A^{n}}=\frac{1}{M_{0}} \sum_{m=1}^{M_{0}=1} \mathcal{E}^{m}\left(\tau_{M_{1}}\right)$ denote the average channel input.

\footnotetext{
${ }^{8} \mathrm{We}$ make this choice for convenience and note that the use of the asymptotic equipartition property (25) would also allow to choose $\varepsilon$ superpolynomially small in $n$.
}

For each fixed $i \in I,\left(M_{0}, M_{1},\left\{\mathcal{E}^{m}\right\},\left\{\Lambda^{m, i}\right\}_{m}\right)$ is a code for entanglement-assisted classical communication through $\left(\mathcal{N}^{i}\right)^{\otimes n}$ with message size $M_{0}$ and error probability $\delta$. Thus, we may apply the converse from [3, Theorem 18, (43) \& Lemma 30],

$$
\log M_{0} \leq \frac{1}{1-\delta}\left(I\left(\rho_{A^{n}},\left(\mathcal{N}^{i}\right)^{\otimes n}\right)+h(\delta)\right),
$$

where we have introduced the notation $I\left(\sigma_{A}, \mathcal{T}\right):=I\left(A^{\prime}\right.$ : $B)_{\mathcal{T}\left[\sigma_{A A^{\prime}}\right]}$, with $\sigma_{A A^{\prime}}$ an arbitrary purification of $\sigma_{A}$. We now use the sub-additivity property [45, (3.24)],

$$
I\left(\rho_{A^{n}},\left(\mathcal{N}^{i}\right)^{\otimes n}\right) \leq \sum_{k=1}^{n} I\left(\rho_{A}^{k}, \mathcal{N}^{i}\right),
$$

where we have defined $\rho_{A}^{k}:=\rho_{A_{k}}$. By definition, the righthand side is equal to

$$
\sum_{k=1}^{n} I\left(\rho_{A}^{k}, \mathcal{N}^{i}\right)=\sum_{k=1}^{n} I\left(A^{\prime}: B\right)_{\mathcal{N}^{i}\left(\rho_{A A^{\prime}}^{k}\right.},
$$

where the $\left|\rho_{A A^{\prime}}^{k}\right\rangle$ denote purifications of the $\rho_{A}^{k}$. To further upper bound this expression, we introduce the pure state

$$
\sigma_{A A^{\prime} X Y}:=\frac{1}{\sqrt{n}} \sum_{k=1}^{n}\left|\rho_{A A^{\prime}}^{k}\right\rangle \otimes|k k\rangle_{X Y} .
$$

Then, $\sigma_{A A^{\prime} X}=\frac{1}{n} \sum_{k=1}^{n} \rho_{A A^{\prime}}^{k} \otimes|k\rangle\left\langle\left. k\right|_{X}\right.$, and therefore

$$
\begin{aligned}
& \frac{1}{n} \sum_{k=1}^{n} I\left(A^{\prime}: B\right)_{\mathcal{N}^{i}\left(\rho_{A A^{\prime}}^{k}\right)}=I\left(A^{\prime}: B \mid X\right)_{\mathcal{N}^{i}(\sigma)} \\
= & I\left(A^{\prime} X: B\right)_{\mathcal{N}^{i}(\sigma)}-I(B: X)_{\mathcal{N}^{i}(\sigma)} \\
\leq & I\left(A^{\prime} X: B\right)_{\mathcal{N}^{i}(\sigma)} \leq I\left(A^{\prime} X Y: B\right)_{\mathcal{N}^{i}(\sigma)} \\
= & I\left(\sigma_{A}, \mathcal{N}^{i}\right),
\end{aligned}
$$

where the first equation uses that $X$ is classical, the second is the chain rule for the conditional mutual information, the third step is the non-negativity of the mutual information, the fourth the monotonicity of mutual information under local quantum operations, and the last equation is again by definition. If we plug (35) into (33) then we obtain the upper bound

$$
\frac{1}{n} \log M_{0} \leq \frac{1}{1-\delta}\left(I\left(\sigma_{A}, \mathcal{N}^{i}\right)+\frac{h(\delta)}{n}\right) .
$$

Crucially, the state $\sigma_{A}=\frac{1}{n} \sum_{k=1}^{n} \rho_{A}^{k}$ does not depend on the channel $\mathcal{N}^{i}$ under consideration (see (34)). Thus, it follows that the above inequality holds for all $i \in I$,

$$
\frac{1}{n} \log M_{0} \leq \frac{1}{1-\delta}\left(\inf _{i \in I} I\left(\sigma_{A}, \mathcal{N}^{i}\right)+\frac{h(\delta)}{n}\right),
$$

and therefore

$$
\begin{aligned}
R & \leq \frac{1}{n} \log M_{0} \\
& \leq \frac{1}{1-\delta}\left(\sup _{\rho} \inf _{i \in I} I\left(\rho_{A}, \mathcal{N}^{i}\right)+\frac{h(\delta)}{n}\right) \\
& =\frac{1}{1-\delta}\left(\sup _{\rho} \inf _{i \in I} I\left(A^{\prime}: B\right)_{\mathcal{N}^{i}(\rho)}+\frac{h(\delta)}{n}\right) .
\end{aligned}
$$

This establishes (32) and thus the claim of the theorem.

As explained at the beginning of Section IV, Lemmas 9 and 10 together establish Theorems 1 and 2 . 


\section{INFORMED SENDER}

\section{A. One-shot coding theorem}

Our one-shot coding theorem in the uninformed scenario can be understood a direct consequence of a corresponding result in [30] for a single fixed channel, applied to the average channel induced by the compound. In the informed sender scenario, such a reduction is complicated by the fact that now the encoders depend on the individual channels in the compound. In the case of the plain quantum capacity, we show how these challenges can in fact be overcome by a suitable reduction, which leads to a pleasant new proof of the corresponding result in [22] (see Section A). In the presence of entanglement assistance, however, we need to develop some new technical tools.

Following the decoupling approach, we start with the following ansatz for the encoders [30]: Given integers $M_{0}$ and $\left\{M_{1}^{i}\right\}_{i=1}^{N}$, let $M_{1}$ denote the least common multiple of the $M_{1}^{i}$. Let $A_{0}$ and $A_{1}$ denote quantum systems of dimensions $M_{0}$ and $M_{1}$, respectively, and fix for each value of $i$ a tensor product decomposition $A_{1} \cong A_{1}^{i} \otimes\left(A_{1}^{i}\right)^{c}$ such that $d_{A_{1}^{i}}=M_{1}^{i}$. Given states $\left\{\rho_{A}^{i}\right\}_{i=1}^{N}$, we now define completely positive maps

$$
\begin{gathered}
\mathcal{E}_{A_{0} A_{1} \rightarrow A}^{i}\left(\sigma_{A_{0} A_{1}}\right):=d_{A} O_{A}\left(\rho^{i}\right) U_{A}^{i} J_{A_{0} A_{1}^{i} \rightarrow A}^{i} \\
\sigma_{A_{0} A_{1}^{i}}\left(J_{A_{0} A_{1}^{i} \rightarrow A}^{i}\right)^{\dagger}\left(U_{A}^{i}\right)^{\dagger}\left(O_{A}\left(\rho^{i}\right)\right)^{\dagger},
\end{gathered}
$$

where the $J_{A_{0} A_{1}^{i} \rightarrow A}^{i}$ are fixed full-rank partial isometries, the $U_{A}^{i}$ denote unitaries that will later be chosen at random, and where we use the notation

$$
O_{A}(\rho):=\sum_{a, a^{\prime}} \rho_{a, a^{\prime}}|a\rangle\left\langle\left. a^{\prime}\right|_{A}\right.
$$

with $\rho_{a, a^{\prime}}$ the coefficients obtained by expanding the pure state $\left|\rho_{A A^{\prime}}\right\rangle$ in the same computational basis as our maximally entangled states, i.e., $\left|\rho_{A A^{\prime}}\right\rangle=\sum_{a, a^{\prime}} \rho_{a, a^{\prime}}\left|a_{A}\right\rangle\left|a_{A^{\prime}}^{\prime}\right\rangle$. Then we have $\left|\rho_{A A^{\prime}}\right\rangle=\sqrt{d_{A}} O_{A}(\rho)\left|\Phi_{A A^{\prime}}^{+}\right\rangle$. We caution that $O_{A}(\rho)$ is not in general Hermitian.

To assess the performance of the encoders $\left\{\mathcal{E}^{i}\right\}_{i=1}^{N}$, we will consider the average encoder-and-channel

$$
\overline{\mathcal{T}}_{A_{0} A_{1} \rightarrow B}:=\frac{1}{N} \sum_{i=1}^{N} \mathcal{N}_{A \rightarrow B}^{i} \circ \mathcal{E}_{A_{0} A_{1} \rightarrow A}^{i}
$$

and show that the complementary map decouples the reference $R$ from the environment. Following the decoupling approach, this will guarantee the existence of an uninformed decoder $\mathcal{D}_{B_{1} B \rightarrow A}$ for the map $\overline{\mathcal{T}}_{A_{0} A_{1} \rightarrow B}$ and therefore, as in Section IV-A above, for each of its branches $\mathcal{N}^{i} \circ \mathcal{E}^{i}$.

For the purposes of obtaining a decoupling result in terms of smooth entropies it will in fact be useful to consider more general maps of the form

$$
\begin{gathered}
\overline{\mathcal{T}}_{A_{0} A_{1} \rightarrow B}\left(\sigma_{A_{0} A_{1}}\right):=\frac{1}{N} \sum_{i=1}^{N} \mathcal{T}_{A \rightarrow B}^{i}\left(U_{A}^{i} J_{A_{0} A_{1}^{i} \rightarrow A}^{i}\right. \\
\left.\sigma_{A_{0} A_{1}^{i}}\left(J_{A_{0} A_{1}^{i} \rightarrow A}^{i}\right)^{\dagger}\left(U_{A}^{i}\right)^{\dagger}\right),
\end{gathered}
$$

where the $\mathcal{T}_{A \rightarrow B}^{i}$ are arbitrary completely positive maps; we recover (38) for the choice

$$
\mathcal{T}_{A \rightarrow B}^{i}\left(\sigma_{A}\right)=\mathcal{N}_{A \rightarrow B}^{i}\left(d_{A} O_{A}\left(\rho^{i}\right) \sigma_{A}\left(O_{A}\left(\rho^{i}\right)\right)^{\dagger}\right) .
$$

We now obtain an explicit complementary map. For this, let $K_{\left(A_{1}^{i}\right)^{c} \rightarrow A^{c}}^{i}$ denote isometries, where $A^{c}$ is an auxiliary system of sufficiently large dimension (e.g., $M_{1}$ ), and let $W_{A \rightarrow B E}^{i}$ denote dilations of the maps $\mathcal{T}_{A \rightarrow B}^{i}$. Then, the maps

$$
\mathcal{T}_{A \rightarrow E}^{i, c}\left(\sigma_{A}\right):=\operatorname{tr}_{B}\left[W_{A \rightarrow B E}^{i} \sigma_{A}\left(W_{A \rightarrow B E}^{i}\right)^{\dagger}\right]
$$

define complementary maps of the channels $\mathcal{T}_{A \rightarrow B}^{i}\left(\sigma_{A}\right)=$ $\operatorname{tr}_{E}\left[W_{A \rightarrow B E}^{i} \sigma_{A}\left(W_{A \rightarrow B E}^{i}\right)^{\dagger}\right]$, and it is not hard to verify that the completely positive map

$$
\begin{gathered}
\overline{\mathcal{T}}_{A_{0} A_{1} \rightarrow A^{c} E I}^{c}\left(\sigma_{A_{0} A_{1}}\right):=\frac{1}{N} \sum_{i, j} \operatorname{tr}_{B}\left[W_{A \rightarrow B E}^{i} U_{A}^{i}\right. \\
J_{A_{0} A_{1}^{i} \rightarrow A^{i}}^{i} K_{\left(A_{1}^{i}\right)^{c} \rightarrow A^{c}}^{i} \sigma_{A_{0} A_{1}}\left(K_{\left(A_{1}^{j}\right)^{c} \rightarrow A^{c}}^{j}\right)^{\dagger} \\
\left.\left(J_{A_{0} A_{1}^{j} \rightarrow A}^{j}\right)^{\dagger}\left(U_{A}^{j}\right)^{\dagger}\left(W_{A \rightarrow B E}^{j}\right)^{\dagger}\right] \otimes|i\rangle\left\langle\left. j\right|_{I}\right.
\end{gathered}
$$

is complementary to the map (39).

Lemma 11. Let $\left\{\mathcal{T}_{A \rightarrow B}^{i}\right\}_{i=1}^{N}$ be completely positive maps, and $M_{0},\left\{M_{1}^{i}\right\}_{i=1}^{N}$ integers such that $M_{0} M_{1}^{i} \leq d_{A}$ for all $i$. Let $\left\{\mathcal{T}_{A \rightarrow E}^{i, c}\right\}_{i=1}^{N}$ and $\overline{\mathcal{T}}_{A_{0} A_{1} \rightarrow A^{c} E I}$ denote the complementary maps as defined in (40) and (41), respectively. Then, we have

$$
\begin{aligned}
& \mathbb{E}\left\|\overline{\mathcal{T}}_{A_{0} A_{1} \rightarrow A^{c} E I}^{c}\left(\Phi_{A_{0} R}^{+} \otimes \tau_{A_{1}}\right)-\omega_{A^{c} E I} \otimes \tau_{R}\right\|_{1} \\
\leq & 2^{-\frac{1}{2}\left(\min _{i} H_{2}\left(A^{\prime} \mid E\right)_{\mathcal{T}^{i, c}(\Phi+)}-\log M_{0}+\log M_{1}^{i}-2 \log N-2\right)},
\end{aligned}
$$

where $\mathbb{E}$ denotes the average over independent Haar-random unitaries $\left\{U_{A}^{i}\right\}$, and

$$
\begin{gathered}
\omega_{A^{c} E I}:=\frac{1}{N} \sum_{i=1}^{N} K_{\left(A_{1}^{i}\right)^{c} \rightarrow A^{c}}^{i} \tau_{\left(A_{1}^{i}\right)^{c}}\left(K_{\left(A_{1}^{i}\right)^{c} \rightarrow A^{c}}^{i}\right)^{\dagger} \\
\otimes \mathcal{T}_{A \rightarrow E}^{i, c}\left(\tau_{A}\right) \otimes|i\rangle\left\langle\left. i\right|_{I} .\right.
\end{gathered}
$$

Proof. We start by bounding the trace norm deviation from the average state by using the triangle inequality:

$$
\begin{gathered}
\mathbb{E}\left\|\overline{\mathcal{T}}_{A_{0} A_{1} \rightarrow A^{c} E I}^{c}\left(\Phi_{A_{0} R}^{+} \otimes \tau_{A_{1}}\right)-\omega_{A^{c} E I} \otimes \tau_{R}\right\|_{1} \\
\leq \frac{1}{N} \sum_{i, j} \mathbb{E} \|\left(\operatorname { t r } _ { B } \left[\left(W^{i} U^{i} J^{i} K^{i}\left(\Phi_{A_{0} R}^{+} \otimes \tau_{A_{1}}\right)\right.\right.\right. \\
\left.-\left(K^{j}\right)^{\dagger}\left(J^{j}\right)^{\dagger}\left(U^{j}\right)^{\dagger}\left(W^{j}\right)^{\dagger}\right] \\
\leq \frac{1}{N} \sum_{i=1}^{N} \mathbb{E} \| \mathcal{T}^{i, c}\left(U^{i} J^{i}\left(\Phi_{A_{0} R}^{+} \otimes \tau_{A_{1}^{i}}\right)\left(J^{i}\right)^{\dagger}\left(U^{i}\right)^{\dagger}\right) \\
-\frac{1}{N} \sum_{i \neq j} \mathbb{E}\left\|\mathcal{T}^{i, c}\left(\tau_{A}^{i}\right) \otimes \tau_{R}\right\|_{A^{c}}\left[W^{i} \|^{\dagger} U^{i} J^{i} K^{i}\left(\Phi_{A_{0} R}^{+} \otimes \tau_{A_{1}}\right)\right. \\
\left.\cdot\left(K^{j}\right)^{\dagger}\left(J^{j}\right)^{\dagger}\left(U^{j}\right)^{\dagger}\left(W^{j}\right)^{\dagger}\right] \|_{1}
\end{gathered}
$$

To bound the averages in (43), we invoke the one-shot decoupling theorem [31, Theorem 3.3] to obtain the first inequality in

$$
\begin{aligned}
& \mathbb{E}\left\|\mathcal{T}^{i, c}\left(U^{i} J^{i}\left(\Phi_{A_{0} R}^{+} \otimes \tau_{A_{1}^{i}}\right)\left(J^{i}\right)^{\dagger}\left(U^{i}\right)^{\dagger}\right)-\mathcal{T}^{i, c}\left(\tau_{A}^{i}\right) \otimes \tau_{R}\right\|_{1} \\
& \leq 2^{-\frac{1}{2}\left(H_{2}\left(A^{\prime} \mid E\right)_{\mathcal{T}^{i, c}(\Phi+)}-\log M_{0}+\log M_{1}^{i}\right)}
\end{aligned}
$$




$$
\begin{aligned}
& \leq \sqrt{\operatorname{tr}\left[\left(\widetilde{\rho}_{A^{\prime} E}^{i}\right)^{2}\right]} \cdot 2^{-\frac{1}{2}\left(-\log M_{0}+\log M_{1}^{i}\right)} \\
& =\sqrt{\operatorname{tr}\left[\left(\widetilde{\rho}_{B}^{i}\right)^{2}\right]} \cdot 2^{-\frac{1}{2}\left(-\log M_{0}+\log M_{1}^{i}\right)}=: x_{i i} .
\end{aligned}
$$

For the second inequality we have defined $\rho_{A^{\prime} B E}^{i}:=W_{A \rightarrow B E}^{i} \Phi_{A A^{\prime}}^{+}\left(W_{A \rightarrow B E}^{i}\right)^{\dagger}$ and $\widetilde{\rho}_{A^{\prime} B E}^{i}:=$ $\left(\alpha_{E}^{i}\right)^{-1 / 4} \rho_{A^{\prime} B E}^{i}\left(\alpha_{E}^{i}\right)^{-1 / 4}$ for an arbitrary choice of state $\alpha_{E}^{i} \in \mathcal{S}(E)$, and used the definition of the conditional collision entropy in (28).

Bounding the averages in (44) is somewhat more involved because we cannot directly rely on previous results. We start with the Hölder inequality [46, Corollary IV.2.6] and obtain the upper bound $\mathbb{E}\left\|\kappa_{A^{c} E R}^{i j}\right\|_{2}$, with $\kappa_{A^{c} B E R}^{i j}$ defined as in (46); here, we have also used that the $K_{\left(A_{1}^{i}\right)^{c} \rightarrow A^{c}}^{i}\left(K_{\left(A_{1}^{i}\right)^{c} \rightarrow A^{c}}^{i}\right)^{\dagger}$ are orthogonal projections onto the ranges of the isometries $K_{\left(A_{1}^{i}\right)^{c} \rightarrow A^{c}}^{i}$. We now apply Jensen's inequality and the swap trick,

$$
\begin{aligned}
& \mathbb{E}\left\|\kappa_{A^{c} E R}^{i j}\right\|_{2} \leq \sqrt{\mathbb{E} \operatorname{tr}\left[\kappa_{A^{c} E R}^{i j}\left(\kappa_{A^{c} E R}^{i j}\right)^{\dagger}\right]} \\
= & \sqrt{\mathbb{E} \operatorname{tr}\left[\left(\kappa_{A^{c} B E R}^{i j} \otimes\left(\kappa_{A^{c} B E R}^{i j}\right)^{\dagger}\right) F_{A^{c} E R}\right]},
\end{aligned}
$$

where we write $F_{S}$ for the operator that swaps two copies of a subsystem $S$ and acts as the identity otherwise. To compute the right-hand side average, it will be useful to introduce the following notation:

$$
\begin{aligned}
& \widetilde{W}_{A \rightarrow B E}^{i}:=\left(\alpha_{E}^{i}\right)^{-1 / 4} W_{A \rightarrow B E}^{i} \\
& \Omega_{A A^{c} R}^{i j}:=J^{i} K^{i}\left(\Phi_{A_{0} R}^{+} \otimes \tau_{A_{1}}\right)\left(K^{j}\right)^{\dagger}\left(J^{j}\right)^{\dagger},
\end{aligned}
$$

so that

$$
\begin{aligned}
\kappa_{A^{c} B E R}^{i j} & =\widetilde{W}_{A \rightarrow B E}^{i} U_{A}^{i} \Omega_{A A^{c} R}^{i j}\left(U_{A}^{j}\right)^{\dagger}\left(\widetilde{W}_{A \rightarrow B E}^{j}\right)^{\dagger} \\
& =\left(\kappa_{A^{c} B E R}^{j i}\right)^{\dagger} .
\end{aligned}
$$

Then, we get

$$
\begin{aligned}
& \mathbb{E} \operatorname{tr}\left[\left(\kappa_{A^{c} B E R}^{i j} \otimes\left(\kappa_{A^{c} B E R}^{i j}\right)^{\dagger}\right) F_{A^{c} E R}\right] \\
= & \mathbb{E} \operatorname{tr}\left[\left(\widetilde{W}_{A \rightarrow B E}^{i} U_{A}^{i} \Omega_{A A^{c} R}^{i j}\left(U_{A}^{j}\right)^{\dagger}\left(\widetilde{W}_{A \rightarrow B E}^{j}\right)^{\dagger}\right.\right. \\
\otimes & \left.\left.\widetilde{W}_{A \rightarrow B E}^{j} U_{A}^{j} \Omega_{A A^{c} R}^{j i}\left(U_{A}^{i}\right)^{\dagger}\left(\widetilde{W}_{A \rightarrow B E}^{i}\right)^{\dagger}\right) F_{A^{c} E R}\right] \\
= & d_{A}^{-2} \sum_{a, b, c, d} \operatorname{tr}\left[\left(\widetilde{W}_{A \rightarrow B E}^{i}|a\rangle\left\langle b\left|\Omega_{A A^{c} R}^{i j}\right| d\right\rangle\langle c|\left(\widetilde{W}_{A \rightarrow B E}^{j}\right)^{\dagger}\right.\right. \\
\otimes & \left.\left.\widetilde{W}_{A \rightarrow B E}^{j}|c\rangle\left\langle d\left|\Omega_{A A^{c} R}^{j i}\right| b\right\rangle\langle a|\left(\widetilde{W}_{A \rightarrow B E}^{i}\right)^{\dagger}\right) F_{A^{c} E R}\right],
\end{aligned}
$$

since $\mathbb{E}\left(U_{A}^{i} \otimes\left(U_{A}^{i}\right)^{\dagger}\right)=d_{A}^{-1} F_{A}=d_{A}^{-1} \sum_{a, b}|a\rangle\langle b|\otimes| b\rangle\langle a|$ and likewise for $U_{A}^{j}$. This in turn is equal to

$$
\begin{aligned}
& d_{A}^{-2} \sum_{a, c} \operatorname{tr} {\left[\left(\widetilde{W}_{A \rightarrow B E}^{i}|a\rangle\langle c|\left(\widetilde{W}_{A \rightarrow B E}^{j}\right)^{\dagger}\right.\right.} \\
& \otimes\left.\left.\widetilde{W}_{A \rightarrow B E}^{j}|c\rangle\langle a|\left(\widetilde{W}_{A \rightarrow B E}^{i}\right)^{\dagger}\right) F_{E}\right] \\
& \cdot \sum_{b, d} \operatorname{tr}\left[\left(\left\langle b\left|\Omega_{A A^{c} R}^{i j}\right| d\right\rangle \otimes\left\langle d\left|\Omega_{A A^{c} R}^{j i}\right| b\right\rangle\right) F_{A^{c} R}\right] \\
&=d_{A}^{-2} \operatorname{tr}\left[\left(\widetilde{W}_{A \rightarrow B E}^{i}\left(\widetilde{W}_{A \rightarrow B E}^{i}\right)^{\dagger} \otimes \widetilde{W}_{A \rightarrow B E}^{j}\left(\widetilde{W}_{A \rightarrow B E}^{j}\right)^{\dagger}\right) F_{B}\right] \\
& \cdot \operatorname{tr}\left[\Omega_{A A^{c} R}^{i j} \Omega_{A A^{c} R}^{j i}\right]
\end{aligned}
$$

$$
\begin{aligned}
& =\operatorname{tr}\left[\left(\widetilde{\rho}_{B E}^{i} \otimes \widetilde{\rho}_{B E}^{j}\right) F_{B}\right] \cdot \operatorname{tr}\left[\left(\Phi_{A_{0} R}^{+} \otimes \tau_{A_{1}}\right)^{2}\right] \\
& =\operatorname{tr}\left[\widetilde{\rho}_{B}^{i} \widetilde{\rho}_{B}^{j}\right] \cdot 2^{-\log M_{1}} .
\end{aligned}
$$

If we combine this result with inequalities (46) and (47), we obtain the following bound:

$$
\begin{aligned}
& \mathbb{E}\left\|\operatorname{tr}_{B}\left[W^{i} U^{i} J^{i} K^{i}\left(\Phi_{A_{0} R}^{+} \otimes \tau_{A_{1}}\right)\left(W^{j} U^{j} J^{j} K^{j}\right)^{\dagger}\right]\right\|_{1} \\
\leq & \sqrt{\operatorname{tr}\left[\widetilde{\rho}_{B}^{i} \widetilde{\rho}_{B}^{j}\right]} \cdot 2^{-\frac{1}{2}\left(-\log M_{0}+\frac{1}{2} \log M_{1}^{i}+\frac{1}{2} \log M_{1}^{j}\right)} \\
= & : x_{i j}
\end{aligned}
$$

We thus obtain the following bound on (42),

$$
\begin{aligned}
& \mathbb{E}\left\|\overline{\mathcal{T}}_{A_{0} A_{1} \rightarrow A^{c} E I}^{c}\left(\Phi_{A_{0} R}^{+} \otimes \tau_{A_{1}}\right)-\omega_{A^{c} E I} \otimes \tau_{R}\right\|_{1} \\
\leq & \frac{1}{N} \sum_{i, j} x_{i j}
\end{aligned}
$$

where the $x_{i j}$ are defined in (45) and (48). At last, we note as in [21, Lemma III.3] that $x_{i j} \leq \sqrt{x_{i i} x_{j j}}$ by the CauchySchwarz inequality, therefore $x_{i j} \leq \max \left(x_{i i}, x_{j j}\right) \leq x_{i i}+x_{j j}$, and that we can thus upper bound

$$
\begin{aligned}
& \frac{1}{N} \sum_{i, j} x_{i j} \leq \sum_{i=1}^{N} x_{i i} \\
= & 2 \sum_{i=1}^{N} \sqrt{\operatorname{tr}\left[\left(\widetilde{\rho}_{A^{\prime} E}^{i}\right)^{2}\right]} \cdot 2^{-\frac{1}{2}\left(-\log M_{0}+\log M_{1}^{i}\right)} \\
\leq & \max _{i} \sqrt{\operatorname{tr}\left[\left(\widetilde{\rho}_{A^{\prime} E}^{i}\right)^{2}\right]} \cdot 2^{-\frac{1}{2}\left(-\log M_{0}+\log M_{1}^{i}-2 \log N-2\right)} .
\end{aligned}
$$

This holds for all choices of $\alpha_{E}^{i}$ in the states $\widetilde{\rho}_{A^{\prime} E}^{i}=$ $\left(\alpha_{E}^{i}\right)^{-1 / 4} \mathcal{N}_{A \rightarrow E}^{i, c}\left(\Phi_{A A^{\prime}}^{+}\right)\left(\alpha_{E}^{i}\right)^{-1 / 4}$. The claimed bound thus follows from the definition of the conditional collision entropy (28).

We now derive a smoothed version of Lemma 11. Later, this will allow us to treat the asymptotic IID limit using the asymptotic equipartition property in the form of (25)-(26). We note that this approach is conceptually different from previous works [21], [22], [23].

Lemma 12. Let $\Pi_{A \rightarrow B}=\left\{\mathcal{N}_{A \rightarrow B}^{i}\right\}_{i=1}^{N}$ be a finite compound channel, $\left\{\rho_{A A^{\prime}}^{i}\right\}_{i=1}^{N}$ pure states, $\left\{\mathcal{E}_{A_{0} A_{1} \rightarrow A}^{i}\right\}_{i=1}^{N}$ the corresponding completely positive maps defined in (36), $M_{0}$, $\left\{M_{1}^{i}\right\}_{i=1}^{N}$ integers such that $M_{0} M_{1}^{i} \leq d_{A}$ for all $i$, and $\varepsilon>0$. Then, there exists a quantum operation $\mathcal{D}_{B B_{1} \rightarrow A_{0}}$ which depends measurably on the random unitaries $\left\{U_{A}^{i}\right\}$ such that

$$
\begin{aligned}
& \mathbb{E} \|\left(\mathcal{D}_{B B_{1} \rightarrow A_{0}} \circ \frac{1}{N} \sum_{i=1}^{N} \mathcal{N}_{A \rightarrow B}^{i} \circ \mathcal{E}_{A_{0} A_{1} \rightarrow A}^{i}\right)\left(\Phi_{A_{0} R}^{+} \otimes \Phi_{A_{1} B_{1}}^{+}\right) \\
& -\Phi_{A_{0} R}^{+} \|_{1} \leq \delta+2 \sqrt{2 \delta}+2 \varepsilon,
\end{aligned}
$$

where

$$
\delta=2^{-\frac{1}{2}\left(-\max _{i} H_{\max }^{\varepsilon}\left(A^{\prime} \mid B\right)_{\mathcal{N}^{i}\left(\rho^{i}\right)}-\log M_{0}+\log M_{1}^{i}-2 \log N-2\right) .}
$$

Proof. According to (24), there exist $\widetilde{\rho}_{A^{\prime} B}^{i} \in S_{\leq}(A B)$ such that

$$
\begin{aligned}
& H_{\max }^{\varepsilon}\left(A^{\prime} \mid B\right)_{\mathcal{N}^{i}\left(\rho^{i}\right)}=H_{\max }\left(A^{\prime} \mid B\right)_{\widetilde{\rho}^{i}} \text { and } \\
& P\left(\mathcal{N}_{A \rightarrow B}^{i}\left(\rho_{A A^{\prime}}^{i}\right), \widetilde{\rho}_{A^{\prime} B}^{i}\right) \leq \varepsilon .
\end{aligned}
$$




$$
\begin{aligned}
& \mathbb{E}\left\|\operatorname{tr}_{B}\left[W^{i} U^{i} J^{i} K^{i}\left(\Phi_{A_{0} R}^{+} \otimes \tau_{A_{1}}\right)\left(K^{j}\right)^{\dagger}\left(J^{j}\right)^{\dagger}\left(U^{j}\right)^{\dagger}\left(W^{j}\right)^{\dagger}\right]\right\|_{1} \\
\leq & \left\|\left(\alpha_{E}^{i} \otimes I_{R} \otimes K_{\left(A_{1}^{i}\right)^{c} \rightarrow A^{c}}^{i}\left(K_{\left(A_{1}^{i}\right)^{c} \rightarrow A^{c}}^{i}\right)^{\dagger}\right)^{1 / 4}\right\|_{4} \cdot \|\left(\alpha_{E}^{j} \otimes I_{R} \otimes K_{\left(A_{1}^{j}\right)^{c} \rightarrow A^{c}}\left(K_{\left(A_{1}^{j}\right)^{c} \rightarrow A^{c}}^{c^{\dagger}}\right)^{1 / 4} \|_{4}\right. \\
& \cdot \mathbb{E}\left\|\left(\alpha_{E}^{i}\right)^{-1 / 4} \operatorname{tr}_{B}\left[W^{i} U^{i} J^{i} K^{i}\left(\Phi_{A_{0} R}^{+} \otimes \tau_{A_{1}}\right)\left(K^{j}\right)^{\dagger}\left(J^{j}\right)^{\dagger}\left(U^{j}\right)^{\dagger}\left(W^{j}\right)^{\dagger}\right]\left(\alpha_{E}^{j}\right)^{-1 / 4}\right\|_{2} \\
= & 2^{-\frac{1}{2}\left(-\log M_{0}+\frac{1}{2} \log M_{1}^{i}+\frac{1}{2} \log M_{1}^{j}-\log M_{1}\right)} \\
& \cdot \mathbb{E}\left\|\operatorname{tr}_{B}[\underbrace{\left(\alpha_{E}^{i}\right)^{-1 / 4} W^{i} U^{i} J^{i} K^{i}\left(\Phi_{A_{0} R}^{+} \otimes \tau_{A_{1}}\right)\left(K^{j}\right)^{\dagger}\left(J^{j}\right)^{\dagger}\left(U^{j}\right)^{\dagger}\left(W^{j}\right)^{\dagger}\left(\alpha_{E}^{j}\right)^{-1 / 4}}_{=: \kappa_{A^{i} B E R}^{i j}}]\right\|_{2},
\end{aligned}
$$

Let $\mathcal{T}_{A \rightarrow B}^{i}$ denote completely positive maps with $\mathcal{T}_{A \rightarrow B}^{i}\left(\Phi_{A A^{\prime}}^{+}\right)=\widetilde{\rho}_{A^{\prime} B}^{i}$ as their Choi-Jamiolkowski states, and define $\left\{\mathcal{T}_{A \rightarrow E}^{i, c}\right\}_{i=1}^{N}, \overline{\mathcal{T}}_{A_{0} A_{1} \rightarrow A}$ and $\overline{\mathcal{T}}_{A \rightarrow A^{c} E I}^{c}$ as in (39)-(41). Then, we have

$$
\begin{aligned}
H_{\text {max }}^{\varepsilon}\left(A^{\prime} \mid B\right)_{\mathcal{N}^{i}\left(\rho^{i}\right)} & =H_{\max }\left(A^{\prime} \mid B\right)_{\mathcal{T}^{i}\left(\Phi^{+}\right)} \\
& =-H_{\min }\left(A^{\prime} \mid E\right)_{\mathcal{T}^{i, c}\left(\Phi^{+}\right)}
\end{aligned}
$$

by (23). For each realization of the random unitaries $\left\{U_{A}^{i}\right\}$, Lemma 5 implies that there exists a quantum operation $\mathcal{D}_{B B_{1} \rightarrow A_{0}}$ (partial isometry) such that

$$
\begin{aligned}
& \left\|\mathcal{D}_{B B_{1} \rightarrow A_{0}} \circ \overline{\mathcal{T}}_{A_{0} A_{1} \rightarrow B}\left(\Phi_{A_{0} R}^{+} \otimes \Phi_{A_{1} B_{1}}^{+}\right)-\Phi_{A_{0} R}^{+}\right\|_{1} \\
& \leq \Delta+2 \sqrt{2 \Delta}
\end{aligned}
$$

where

$$
\Delta:=\left\|\overline{\mathcal{T}}_{A_{0} A_{1} \rightarrow A^{c} E I}^{c}\left(\Phi_{A_{0} R}^{+} \otimes \tau_{A_{1}}\right)-\omega_{A^{c} E I} \otimes \tau_{R}\right\|_{1},
$$

with $\omega_{A^{c} E I}$ as defined in Lemma 11. In fact, $\mathcal{D}_{B B_{1} \rightarrow A_{0}}$ can be chosen as a measurable function of the $\left\{U_{A}^{i}\right\}$, so that it can itself be regarded as a random variable. Thus, it makes sense to bound the following expression:

$$
\begin{aligned}
& \mathbb{E}\left\|\left(\mathcal{D} \circ \frac{1}{N} \sum_{i=1}^{N} \mathcal{N}^{i} \circ \mathcal{E}^{i}\right)\left(\Phi_{A_{0} R}^{+} \otimes \Phi_{A_{1} B_{1}}^{+}\right)-\Phi_{A_{0} R}^{+}\right\|_{1} \\
& \leq \frac{1}{N} \sum_{i=1}^{N} \mathbb{E} \|\left(\widehat{\mathcal{T}}^{i}-\mathcal{T}^{i}\right)\left(U^{i} J^{i}\left(\Phi_{A_{0} R}^{+} \otimes \Phi_{A_{1}^{i} B_{1}^{i}}^{+}\right)\right. \\
& \left.\left(J^{i}\right)^{\dagger}\left(U_{A}^{i}\right)^{\dagger}\right) \|_{1} \\
& +\mathbb{E} \| \mathcal{D}_{B B_{1} \rightarrow A_{0}} \circ \overline{\mathcal{T}}_{A_{0} A_{1} \rightarrow B}\left(\Phi_{A_{0} R}^{+} \otimes \Phi_{A_{1} B_{1}}^{+}\right) \\
& \quad-\Phi_{A_{0} R}^{+} \|_{1},
\end{aligned}
$$

where $\widehat{\mathcal{T}}_{A \rightarrow B}^{i}\left(\sigma_{A}\right):=\mathcal{N}_{A \rightarrow B}^{i}\left(d_{A} O_{A}\left(\rho^{i}\right) \sigma_{A} O_{A}\left(\rho^{i}\right)^{\dagger}\right)$. In the first inequality, we have inserted (36)-(39) and used the triangle inequality as well as that $\mathcal{D}_{B B_{1} \rightarrow A_{0}}$ is tracenonincreasing.

To bound the averages in (53), we now follow the smoothing ideas from [31]. Let $\widehat{\rho}_{A^{\prime} B}^{i}:=\widehat{\mathcal{T}}_{A \rightarrow B}^{i}\left(\Phi_{A A^{\prime}}^{+}\right)=\mathcal{N}_{A \rightarrow B}^{i}\left(\rho_{A A^{\prime}}^{i}\right)$ and write $\widehat{\rho}_{A^{\prime} B}^{i}-\widetilde{\rho}_{A^{\prime} B}^{i}=\delta_{A^{\prime} B}^{i,+}-\delta_{A^{\prime} B}^{i,}$ as a difference of positive semidefinite operators, so that

$$
\begin{aligned}
& \operatorname{tr}\left[\delta_{A^{\prime} B}^{i+}\right]+\operatorname{tr}\left[\delta_{A^{\prime} B}^{i,-}\right]=\left\|\widehat{\rho}_{A^{\prime} B}^{i}-\widetilde{\rho}_{A^{\prime} B}^{i}\right\|_{1} \\
\leq & 2 P\left(\widehat{\rho}_{A^{\prime} B}^{i}, \widetilde{\rho}_{A^{\prime} B}^{i}\right) \leq 2 \varepsilon
\end{aligned}
$$

by (17) together with (49). Let $\mathcal{D}_{A \rightarrow B}^{i, \pm}$ denote completely positive maps whose Choi-Jamiolkowski states are $\delta_{A^{\prime} B}^{i, \pm}$, respectively. Then, we have $\widehat{\mathcal{T}}_{A \rightarrow B}^{i}-\mathcal{T}_{A \rightarrow B}^{i}=\mathcal{D}_{A \rightarrow B}^{i,+}-\mathcal{D}_{A \rightarrow B}^{i,-}$, and hence

$$
\begin{aligned}
& \mathbb{E}\left\|\left(\widehat{\mathcal{T}}_{A \rightarrow B}^{i}-\mathcal{T}_{A \rightarrow B}^{i}\right)\left(U^{i} J^{i}\left(\Phi_{A_{0} R}^{+} \otimes \Phi_{A_{1}^{i} B_{1}^{i}}^{+}\right)\left(J^{i}\right)^{\dagger}\left(U^{i}\right)^{\dagger}\right)\right\|_{1} \\
\leq & \mathbb{E} \operatorname{tr}\left[\mathcal{D}_{A \rightarrow B}^{i,+}\left(U_{A}^{i} J^{i}\left(\Phi_{A_{0} R}^{+} \otimes \Phi_{A_{1}^{i} B_{1}^{i}}^{+}\right)\left(J^{i}\right)^{\dagger}\left(U_{A}^{i}\right)^{\dagger}\right)\right] \\
& +\mathbb{E} \operatorname{tr}\left[\mathcal{D}_{A \rightarrow B}^{i,-}\left(U_{A}^{i} J^{i}\left(\Phi_{A_{0} R}^{+} \otimes \Phi_{A_{1}^{i} B_{1}^{i}}^{+}\right)\left(J^{i}\right)^{\dagger}\left(U_{A}^{i}\right)^{\dagger}\right)\right] \\
\leq & \operatorname{tr}\left[\delta_{B}^{i,+} \otimes \tau_{R B_{1}^{i}}\right]+\operatorname{tr}\left[\delta_{B}^{i,-} \otimes \tau_{R B_{1}^{i}}\right] \\
\leq & 2 \varepsilon
\end{aligned}
$$

by the triangle inequality, the fact that $\mathbb{E}\left(U_{A}^{i} \sigma_{A B}\left(U_{A}^{i}\right)^{\dagger}\right)=$ $\tau_{A} \otimes \operatorname{tr}_{A}\left[\sigma_{A B}\right]$ for all $\sigma_{A B}$, and (55).

To bound the average in (54), we use (51), Jensen's inequality and Lemma 11 to obtain

$$
\begin{aligned}
& \mathbb{E}\left\|\mathcal{D}_{B B_{1} \rightarrow A_{0}} \circ \overline{\mathcal{T}}_{A_{0} A_{1} \rightarrow B}\left(\Phi_{A_{0} R}^{+} \otimes \Phi_{A_{1} B_{1}}^{+}\right)-\Phi_{A_{0} R}^{+}\right\|_{1} \\
\leq & (\mathbb{E} \Delta)+2 \sqrt{2 \mathbb{E} \Delta} \leq \delta+2 \sqrt{2 \delta}
\end{aligned}
$$

with

$$
\delta=2^{-\frac{1}{2}\left(-\max _{i} H_{\max }^{\varepsilon}\left(A^{\prime} \mid B\right)_{\mathcal{N}^{i}\left(\rho^{i}\right)}-\log M_{0}+\log M_{1}^{i}-2 \log N-2\right),}
$$

where we used that $H_{2}\left(A^{\prime} \mid E\right)_{\mathcal{T}^{i, c}\left(\Phi^{+}\right)} \geq H_{\min }\left(A^{\prime} \mid E\right)_{\mathcal{T}^{i, c}\left(\Phi^{+}\right)}$ and (50). Plugging both bounds into (52), we obtain the desired estimate.

The decoding maps $\mathcal{E}_{A_{0} A_{1} \rightarrow A}^{i}$ as defined in (36) are completely positive but not in general trace-preserving, and therefore not valid quantum operations. However, the following lemma, whose proof is entailed in [30, Theorem 3.14] and which can be deduced directly from the one-shot decoupling theorem [31, Theorem 3.3], will later allow us to replace the $\mathcal{E}^{i}$ by valid quantum operations.

Lemma 13. Let $\mathcal{E}_{A_{0} A_{1} \rightarrow A}^{i}$ be one of the completely positive maps defined in (36) for some state $\rho_{A}^{i}$. Then, we have

$$
\begin{aligned}
& \mathbb{E}\left\|\operatorname{tr}_{A}\left[\mathcal{E}_{A_{0} A_{1} \rightarrow A}^{i}\left(\Phi_{A_{0} R}^{+} \otimes \Phi_{A_{1} B_{1}}^{+}\right)\right]-\tau_{R B_{1}}\right\|_{1} \\
\leq & 2^{-\frac{1}{2}\left(H_{\min }^{\varepsilon}(A)_{\rho^{i}}-\log M_{0}-\log M_{1}^{i}\right)}+12 \varepsilon
\end{aligned}
$$

By combining this with Lemma 12, we obtain our one-shot coding theorem for compound channels in the informed sender scenario.

Theorem 14. Let $\Pi_{A \rightarrow B}=\left\{\mathcal{N}_{A \rightarrow B}^{i}\right\}_{i=1}^{N}$ be a finite compound channel, $\left\{\rho_{A A^{\prime}}^{i}\right\}_{i=1}^{N}$ pure states, and $M_{0},\left\{M_{1}^{i}\right\}_{i=1}^{N}$ integers 
such that $M_{0} M_{1}^{i} \leq d_{A}$ for all $i$, and $\varepsilon \in(0,1]$. Then, there exist quantum operations $\mathcal{E}_{A_{0} A_{1} \rightarrow A}$ and $\mathcal{D}_{B B_{1} \rightarrow A_{0}}$, where $d_{A_{0}}=M_{0}$ and $d_{A_{1}}=d_{B_{1}} \geq \max _{i} M_{1}^{i}$, such that

$$
\begin{aligned}
& \min _{i} F\left(\Phi_{A_{0} R}^{+}, \mathcal{D} \circ \mathcal{N}^{i} \circ \mathcal{E}^{i}\left(\Phi_{A_{0} R}^{+} \otimes \Phi_{A_{1} B_{1}}^{+}\right)\right) \\
\geq & 1-8 N(N+2)\left(\sqrt{\delta_{1}}+\sqrt{\delta_{2}}+6 \sqrt{\varepsilon}\right),
\end{aligned}
$$

where

$$
\begin{aligned}
& \delta_{1}=\max _{i} 2^{-\frac{1}{2}\left(H_{\min }^{\varepsilon}(A)_{\rho^{i}}-\log M_{0}-\log M_{1}^{i}\right)}, \\
& \delta_{2}=\max _{i} 2^{-\frac{1}{2}\left(-H_{\max }^{\varepsilon}\left(A^{\prime} \mid B\right)_{\mathcal{N}^{i}\left(\rho^{i}\right)}-\log M_{0}+\log M_{1}^{i}-2 \log N-2\right) .}
\end{aligned}
$$

Proof. We choose $M_{1}$ as the least common multiple of the $M_{1}^{i}$. By Lemmas 12 and 13, Markov's inequality $\operatorname{Prob}(Z>$ $k \mathbb{E}(Z)) \leq 1 / k$ (for $k=N+2$ ), and the union bound, there exist unitaries $\left\{U_{A}^{i}\right\}_{i=1}^{N}$ and a quantum operation $\mathcal{D}_{B B_{1} \rightarrow A_{0}}$ such that

$$
\begin{aligned}
& \left\|\operatorname{tr}_{A}\left[\mathcal{E}_{A_{0} A_{1} \rightarrow A}^{i}\left(\Phi_{A_{0} R}^{+} \otimes \Phi_{A_{1} B_{1}}^{+}\right)\right]-\tau_{R B_{1}}\right\|_{1} \\
\leq & (N+2)\left(\delta_{1}+12 \varepsilon\right) \quad(\forall i=1, \ldots, N)
\end{aligned}
$$

with

$$
\begin{aligned}
& \|\left(\mathcal{D}_{B B_{1} \rightarrow A_{0}} \circ \frac{1}{N} \sum_{i=1}^{N} \mathcal{N}_{A \rightarrow B}^{i} \circ \mathcal{E}_{A_{0} A_{1} \rightarrow A}^{i}\right)\left(\Phi_{A_{0} R}^{+} \otimes \Phi_{A_{1} B_{1}}^{+}\right) \\
& -\Phi_{A_{0} R}^{+} \|_{1} \leq(N+2)\left(\delta_{2}+2 \sqrt{2 \delta_{2}}+2 \varepsilon\right) .
\end{aligned}
$$

As a consequence of the first bound and Lemma 5, we can find quantum operations $\left\{\widetilde{\mathcal{E}}_{A_{0} A_{1} \rightarrow A}^{i}\right\}_{i=1}^{N}$ such that

$$
\begin{aligned}
& \left\|\left(\widetilde{\mathcal{E}}_{A_{0} A_{1} \rightarrow A}^{i}-\mathcal{E}_{A_{0} A_{1} \rightarrow A}^{i}\right)\left(\Phi_{A_{0} R}^{+} \otimes \Phi_{A_{1} B_{1}}^{+}\right)\right\|_{1} \\
\leq & (N+2)\left(\delta_{1}+12 \varepsilon\right)+2 \sqrt{2(N+2)\left(\delta_{1}+12 \varepsilon\right)} .
\end{aligned}
$$

Now, using the triangle inequality as well as the fact that $\mathcal{D}$ and the $\mathcal{N}^{i}$ are completely positive and trace-nonincreasing, we obtain that

$$
\begin{aligned}
& \left\|\frac{1}{N} \sum_{i=1}^{N}\left(\mathcal{D} \circ \mathcal{N}^{i} \circ \widetilde{\mathcal{E}}^{i}\right)\left(\Phi_{A_{0} R}^{+} \otimes \Phi_{A_{1} B_{1}}^{+}\right)-\Phi_{A_{0} R}^{+}\right\|_{1} \\
\leq & \frac{1}{N} \sum_{i=1}^{N}\left\|\left(\widetilde{\mathcal{E}}_{A_{0} A_{1} \rightarrow A}^{i}-\mathcal{E}_{A_{0} A_{1} \rightarrow A}^{i}\right)\left(\Phi_{A_{0} R}^{+} \otimes \Phi_{A_{1} B_{1}}^{+}\right)\right\|_{1} \\
& +\left\|\left(\mathcal{D} \circ \frac{1}{N} \sum_{i=1}^{N} \mathcal{N}^{i} \circ \mathcal{E}^{i}\right)\left(\Phi_{A_{0} R}^{+} \otimes \Phi_{A_{1} B_{1}}^{+}\right)-\Phi_{A_{0} R}^{+}\right\|_{1} \\
\leq & (N+2)\left(\delta_{1}+12 \varepsilon\right)+2 \sqrt{2(N+2)\left(\delta_{1}+12 \varepsilon\right)} \\
& +(N+2)\left(\delta_{2}+2 \sqrt{2 \delta_{2}}+2 \varepsilon\right) \\
\leq & 4(N+2)\left(\sqrt{\delta_{1}}+\sqrt{\delta_{2}}+6 \sqrt{\varepsilon}\right) .
\end{aligned}
$$

To arrive at the last inequality, we have assumed that $\delta_{1,2} \leq 1$ (without loss of generality, since the bound (56) is otherwise vacuous). At last, we use (15) to turn this into a lower bound on the average entanglement fidelity:

$$
\begin{aligned}
& F\left(\Phi_{A_{0} R}^{+},\left(\frac{1}{N} \sum_{i=1}^{N} \mathcal{D} \circ \mathcal{N}^{i} \circ \widetilde{\mathcal{E}}^{i}\right)\left(\Phi_{A_{0} R}^{+} \otimes \Phi_{A_{1} B_{1}}^{+}\right)\right) \\
\geq & 1-8(N+2)\left(\sqrt{\delta_{1}}+\sqrt{\delta_{2}}+6 \sqrt{\varepsilon}\right) .
\end{aligned}
$$

Using the same argument that we used to derive (29), this implies that

$$
\begin{aligned}
& \min _{i} F\left(\Phi_{A_{0} R}^{+},\left(\mathcal{D} \circ \mathcal{N}^{i} \circ \widetilde{\mathcal{E}}^{i}\right)\left(\Phi_{A_{0} R}^{+} \otimes \Phi_{A_{1} B_{1}}^{+}\right)\right) \\
\geq & 1-8 N(N+2)\left(\sqrt{\delta_{1}}+\sqrt{\delta_{2}}+6 \sqrt{\varepsilon}\right) .
\end{aligned}
$$

\section{B. Achievability}

Given the one-shot coding theorem phrased in terms of smooth entropies (Theorem 14), we can now prove the direct part of Theorem 3 in a similar fashion as for Theorem 1:

Lemma 15. Let $\Pi_{A \rightarrow B}=\left\{\mathcal{N}_{A \rightarrow B}^{i}\right\}_{i \in I}$ be a compound channel with arbitrary index set I. Then, we have

$$
Q_{E, I S}\left(\Pi_{A \rightarrow B}\right) \geq \inf _{i \in I} Q_{E}\left(\mathcal{N}_{A \rightarrow B}^{i}\right) .
$$

Proof. Let $\Delta>0$ and

$$
R=\inf _{i \in I} Q_{E}\left(\mathcal{N}_{A \rightarrow B}^{i}\right)-\Delta .
$$

We will show that for any $\delta>0$ there exists $n_{0}$ such that, for all $n \geq n_{0}$, the triple $(R, n, \delta)$ is achievable for $\Pi_{A \rightarrow B}$. If $R \leq 0$ then there is nothing to show, thus we may assume that $R>0$.

As in the proof of Lemma 9, we will use the discretization result [21, Lemma V.2] to reduce to finite compound channels. It shows that for any $n$ there exists a finite compound channel $\widetilde{\Pi}_{A \rightarrow B}=\left\{\widetilde{\mathcal{N}}_{A \rightarrow B}^{j}\right\}_{j=1}^{N}$ of cardinality $N \leq(3 n)^{4 d_{A B}^{2}}$ with the property that for any $\mathcal{N}^{i} \in \Pi$ there exists some $\widetilde{\mathcal{N}}^{j} \in \widetilde{\Pi}$ such that $\left\|\left(\mathcal{N}^{i}\right)^{\otimes k}-\left(\tilde{\mathcal{N}}^{j}\right)^{\otimes k}\right\|_{\diamond} \leq k / n^{2}$ for all $k$, and vice versa. In particular, for $k=1$ this bound together with (20) implies that

$$
\begin{aligned}
& \left|\inf _{i \in I} Q_{E}\left(\mathcal{N}^{i}\right)-\min _{j=1, \ldots, N} Q_{E}\left(\tilde{\mathcal{N}}^{j}\right)\right| \\
\leq & \frac{1}{n^{2}} \log \min \left\{d_{A}, d_{B}\right\}+2\left(1+\frac{1}{2 n^{2}}\right) h\left(\frac{1}{1+2 n^{2}}\right) .
\end{aligned}
$$

As a consequence,

$$
R \leq \min _{j=1, \ldots, N} Q_{E}\left(\tilde{\mathcal{N}}^{j}\right)-\frac{\Delta}{2}
$$

for $n$ sufficiently large (depending only on $d_{A B}$ and $\Delta$ ). Let us assume that this is the case.

Let $\left\{\rho_{A A^{\prime}}^{j}\right\}$ be pure states such that $Q_{E}\left(\widetilde{\mathcal{N}}^{j}\right)=\frac{1}{2} I\left(A^{\prime}\right.$ : $B)_{\widetilde{\mathcal{N}}^{j}\left(\rho^{j}\right)}$ for all $j=1, \ldots, N$. We now apply our one-shot coding result, Theorem 14, to $\widetilde{\Pi}_{A \rightarrow B}^{\otimes n}=\left\{\left(\widetilde{\mathcal{N}}_{A \rightarrow B}^{j}\right)^{\otimes n}\right\}_{j=1}^{N}$, $\left\{\left(\rho_{A A^{\prime}}^{j}\right)^{\otimes n}\right\}_{j=1}^{N}, M_{0}=\left\lceil 2^{n R}\right\rceil, M_{1}^{j}=\left\lceil 2^{n\left(H(A) \rho_{\rho^{j}}-R-\Delta / 2\right)}\right\rceil$. We note that

$$
\begin{aligned}
M_{0} M_{1}^{j} & \leq 2^{n R+1} 2^{n\left(H(A)_{\rho^{j}}-R-\Delta / 2\right)+1} \\
& =2^{n\left(H(A)_{\rho^{j}}-\Delta / 2+2 / n\right)} \leq d_{A^{n}}
\end{aligned}
$$

for $n$ sufficiently large (depending only on $R$ and $\Delta$ ). Thus, the assumption on the integers $M_{0},\left\{M_{1}^{j}\right\}$ is satisfied. For all $\varepsilon \in(0,1]$, we obtain encoders $\widetilde{\mathcal{E}}_{A_{0} A_{1} \rightarrow A^{n}}^{j}$ and a decoder $\mathcal{D}_{B^{n} B_{1} \rightarrow A_{0}}$ such that

$$
\begin{aligned}
& \min _{j} F\left(\Phi_{A_{0} R}^{+}, \mathcal{D} \circ\left(\widetilde{\mathcal{N}}^{j}\right)^{\otimes n} \circ \widetilde{\mathcal{E}}^{j}\left(\Phi_{A_{0} R}^{+} \otimes \Phi_{A_{1} B_{1}}^{+}\right)\right) \\
\geq & 1-8 N(N+2)\left(\sqrt{\delta_{1}}+\sqrt{\delta_{2}}+6 \sqrt{\varepsilon}\right),
\end{aligned}
$$


where

$\delta_{1}=\max _{j} 2^{-\frac{1}{2}\left(H_{\min }^{\varepsilon}(A)_{\left(\rho^{j}\right) \otimes n}-\log M_{0}-\log M_{1}^{j}\right)}$,

$\delta_{2}=\max _{j} 2^{-\frac{1}{2}\left(-H_{\max }^{\varepsilon}\left(A^{\prime} \mid B\right)_{\left(\widetilde{\mathcal{N}}^{j}\left(\rho^{j}\right)\right) \otimes n}-\log M_{0}+\log M_{1}^{j}-2 \log N-2\right)}$

We now choose $\varepsilon=1 /(n N)^{4}$. Since $\varepsilon$ decays only polynomially with $n$, the asymptotic equipartition property (25) together with (27) and the estimate (57) implies that

$$
\delta_{1} \leq 2^{-\frac{n}{2}\left(\frac{\Delta}{2}-\frac{2}{n}-\frac{\delta\left(\varepsilon, \rho^{j}\right)}{\sqrt{n}}\right)} \leq 2^{-n \frac{\Delta}{8}} \leq \varepsilon
$$

for sufficiently large $n$ (depending only $d_{A B}, \Delta$ and $R$ ). Likewise, using (26) instead of (25) we obtain that

$$
\delta_{2} \leq 2 N \cdot 2^{-\frac{n}{2}\left(\frac{\Delta}{2}-\frac{1}{n}-\frac{\delta\left(\varepsilon, \widetilde{\mathcal{N}}^{j}\left(\rho^{j}\right)\right)}{\sqrt{n}}\right)} \leq 2 N \cdot 2^{-n \frac{\Delta}{8}} \leq \varepsilon,
$$

where we use that $N$ grows only polynomially with $n$. By inserting the two bounds into (58), we obtain that

$$
\begin{aligned}
& F\left(\Phi_{A_{0} R}^{+}, \mathcal{D} \circ\left(\tilde{\mathcal{N}}^{j}\right) \otimes n \circ \widetilde{\mathcal{E}}^{j}\left(\Phi_{A_{0} R}^{+} \otimes \Phi_{A_{1} B_{1}}^{+}\right)\right) \\
\geq & 1-64 N(N+2) \sqrt{\varepsilon} \geq 1-\frac{192}{n^{2}} .
\end{aligned}
$$

At last, we relate this to the entanglement fidelity for the original compound channel. For this, recall that for any $\mathcal{N}^{i} \in \Pi$ there exists some $\widetilde{\mathcal{N}}^{j} \in \widetilde{\Pi}$ such that $\left\|\left(\mathcal{N}^{i}\right)^{\otimes n}-\left(\widetilde{\mathcal{N}}^{j}\right)^{\otimes n}\right\|_{\diamond} \leq$ $1 / n$. If we choose the encoders correspondingly as $\mathcal{E}^{i}:=\widetilde{\mathcal{E}}^{j}$ then we find using (18) that

$$
\begin{aligned}
& \inf _{i \in I} F\left(\Phi_{A_{0} R}^{+}, \mathcal{D} \circ\left(\mathcal{N}^{i}\right)^{\otimes n} \circ \mathcal{E}^{i}\left(\Phi_{A_{0} R}^{+} \otimes \Phi_{A_{1} B_{1}}^{+}\right)\right) \\
\geq & 1-\frac{192}{n^{2}}-\frac{2}{\sqrt{n}}
\end{aligned}
$$

We conclude that, for any $\delta>0$ and sufficiently large $n$, $(R, \delta, n)$ is a valid triple for the compound channel $\Pi_{A \rightarrow B}$.

\section{Converse}

Since a code for the compound quantum channel $\Pi_{A \rightarrow B}=$ $\left\{\mathcal{N}_{A \rightarrow B}^{i}\right\}$ by definition gives rise to codes for each of its constituent channels $\mathcal{N}_{A \rightarrow B}^{i}$, it is immediate that $Q_{E, I S}(\Pi) \leq$ $Q_{E}\left(\mathcal{N}^{i}\right)$ for all $i \in I$. Thus, we immediately obtain the converse bound in Theorem 3 .

Lemma 16. Let $\Pi_{A \rightarrow B}=\left\{\mathcal{N}_{A \rightarrow B}^{i}\right\}_{i \in I}$ be a compound channel with arbitrary index set $I$. Then, we have

$$
Q_{E, I S}\left(\Pi_{A \rightarrow B}\right) \leq \inf _{i \in I} Q_{E}\left(\mathcal{N}_{A \rightarrow B}^{i}\right)
$$

\section{Feedback ASSistance}

It is well-known that feedback does not increase the entanglement-assisted quantum capacity of a quantum channel [14]. Since any feedback-assisted code for the compound channel gives rise to feedback-assisted codes for each of its constituent channels, we obtain just as in the preceding section the converse bound in Theorem 4. In fact, this holds for arbitrary compound channels:

Lemma 17. Let $\Pi_{A \rightarrow B}=\left\{\mathcal{N}_{A \rightarrow B}^{i}\right\}_{i \in I}$ be a compound channel with arbitrary index set. Then, we have

$$
Q_{E, F}\left(\Pi_{A \rightarrow B}\right) \leq \inf _{i \in I} Q_{E}\left(\mathcal{N}_{A \rightarrow B}^{i}\right) .
$$

Next we show that the upper bound in Lemma 17 is also achievable, at least for finite compound channels (establishing Theorem 4):

Lemma 18. Let $\Pi_{A \rightarrow B}=\left\{\mathcal{N}_{A \rightarrow B}^{i}\right\}_{i \in I}$ be a compound channel with finite index set $|I|<\infty$. Then, we have

$$
Q_{E, F}\left(\Pi_{A \rightarrow B}\right) \geq \inf _{i \in I} Q_{E}\left(\mathcal{N}_{A \rightarrow B}^{i}\right) .
$$

The proof is a generalization of the original proof for classical channels [7] and based on the following quantum channel estimation technique from [24]:

Proposition 19 ([21, Theorem 4.2]). Let $\Pi_{A \rightarrow B}=$ $\left\{\mathcal{N}_{A \rightarrow B}^{i}\right\}_{i=1}^{N}$ be a finite compound channel and set $L=\left(\begin{array}{c}N \\ 2\end{array}\right)$. Then, there exists $f \in(0,1)$ such that for each $m \in \mathbb{N}$ there are mutually orthogonal projectors $\left\{P_{B^{m L}}^{i}\right\}_{i=1}^{N}$ on $B^{\otimes(m L)}$ with $\sum_{i=1}^{N} P_{B^{m L}}^{i}=I_{B^{m L}}$, as well as a pure state $\omega_{A^{m L}}$ on $A^{\otimes(m L)}$ with the property that

$$
\operatorname{tr}\left[P_{B^{m L}}^{i}\left(\mathcal{N}_{A \rightarrow B}^{i}\right)^{\otimes(m L)}\left(\omega_{A^{m L}}\right)\right] \geq\left(1-N f^{m}\right)^{N-1}
$$

for all $i=1, \ldots, N$.

For the IID compound $\Pi_{A \rightarrow B}^{\otimes n}=\left\{\left(\mathcal{N}_{A \rightarrow B}^{i}\right)^{\otimes n}\right\}_{i \in I}$ we use the first $\sqrt{n}$ channel uses to estimate the channel on the receiver's side with the help of Proposition 19. Then, we use feedback to transfer the estimated channel index $i \in I$ to the sender. This allows to use the informed sender protocol for the remaining $n-\sqrt{n}$ channel uses and leads to the same capacity as in the informed sender case. We formalize this strategy in the following proof, which is inspired by [21, Lemma 4.3]:

Proof of Lemma 18. We give a protocol for the IID average channel

$$
\bar{\Pi}_{A^{n} \rightarrow B^{n}}:=\frac{1}{|I|} \sum_{i \in I}\left(\mathcal{N}_{A \rightarrow B}^{i}\right)^{\otimes n},
$$

which will work equally well for the IID compound channel (up to a constant factor of $|I|$ in fidelity, cf. (29)). Take $n=m L+t$ with $L:=\left(\begin{array}{c}|I| \\ 2\end{array}\right)$ and $m \in \mathbb{N}$. We use the first $m L$ instances for channel estimation and the subsequent $t=n-m L$ instances for the actual entanglement transmission, making use of the informed sender protocol as described in Theorem 14.

The encoder $\tilde{\mathcal{E}}_{A^{m L}}$ for the channel estimation inputs $\omega_{A^{m L}}$ from Proposition 19 to the $(m L)$-fold channel. The decoder $\tilde{\mathcal{D}}_{B^{m L}}$ for the channel estimation measures the channel's output as in Proposition 19,

$$
\tilde{\mathcal{D}}_{B^{m L}}(\cdot):=\sum_{i \in I} \tilde{\mathcal{D}}_{B^{m L}}^{i}(\cdot)|i\rangle\left\langle\left. i\right|_{X_{B}}\right.
$$

with $\tilde{\mathcal{D}}_{B^{m L}}^{i}(\cdot):=\operatorname{tr}\left[P_{B^{m L}}^{i}(\cdot)\right]$, and sends the classical outcome $i \in I$ back to the sender, where it is then labeled by the system $X_{A}$. Given this information $i \in I$ we use for the entanglement transmission the informed encoder $\mathcal{E}_{A_{0} A_{1} \rightarrow A^{t}}^{i}$ for $\left(\mathcal{N}_{A \rightarrow B}^{i}\right)^{\otimes t}$, i.e.,

$$
\mathcal{E}_{A_{0} A_{1} X_{A} \rightarrow A^{t}}(\cdot):=\sum_{i \in I} \mathcal{E}_{A_{0} A_{1} \rightarrow A^{t}}^{i}(\cdot) \otimes\langle i|\cdot| i\rangle_{X_{A}},
$$


and the universal decoder $\mathcal{D}_{B^{t} B_{1} \rightarrow A_{0}}$ from Theorem $14 .{ }^{9}$ The total fidelity of the protocol can then be bounded as

$$
\begin{aligned}
& \sum_{i \in I} F\left(\Phi_{A_{0} R}^{+},\left(\left(\tilde{\mathcal{D}}_{B^{m L}}^{i} \otimes \mathcal{D}_{B^{t} B_{1} \rightarrow A_{0}}\right) \circ \bar{\Pi}_{A^{m L+t} \rightarrow B^{m L+t}}\right.\right. \\
& \left.\left.\circ\left(\tilde{\mathcal{E}}_{A^{m L}} \otimes \mathcal{E}_{A_{0} A_{1} \rightarrow A^{t}}^{i}\right)\right)\left(\Phi_{A_{0} R}^{+} \otimes \Phi_{A_{1} B_{1}}^{+}\right)\right) \\
& \geq \frac{1}{|I|} \sum_{i \in I} F\left(\Phi_{A_{0} R}^{+},\left(\left(\tilde{\mathcal{D}}_{B^{m L}}^{i} \otimes \mathcal{D}_{B^{t} B_{1} \rightarrow A_{0}}\right) \circ\left(\mathcal{N}_{A \rightarrow B}^{i}\right)^{\otimes(m L+t)}\right.\right. \\
& \left.\left.\circ\left(\tilde{\mathcal{E}}_{A^{m L} L} \otimes \mathcal{E}_{A_{0} A_{1} \rightarrow A^{t}}^{i}\right)\right)\left(\Phi_{A_{0} R}^{+} \otimes \Phi_{A_{1} B_{1}}^{+}\right)\right) \\
& =\frac{1}{|I|} \sum_{i \in I} F\left(\Phi_{A_{0} R}^{+},\left(\mathcal{D}_{B^{t} B_{1} \rightarrow A_{0}} \circ\left(\mathcal{N}_{A \rightarrow B}^{i}\right)^{\otimes t}\right.\right. \\
& \left.\left.\circ \mathcal{E}_{A_{0} A_{1} \rightarrow A^{t}}^{i}\right)\left(\Phi_{A_{0} R}^{+} \otimes \Phi_{A_{1} B_{1}}^{+}\right)\right) \\
& \cdot \operatorname{tr}\left[P_{B^{m L}}^{i}\left(\mathcal{N}_{A \rightarrow B}^{i}\right)^{\otimes(m L)}\left(\omega_{A^{m L}}\right)\right] \\
& \geq\left(1-|I| f^{m}\right)^{|I|-1}(1-\delta),
\end{aligned}
$$

for $f \in(0,1)$ as in Proposition 19. In the last inequality we have assumed that the informed encoders $\left\{\mathcal{E}_{A_{0} A_{1} \rightarrow A^{t}}^{i}\right\}_{i \in I}$ together with the universal decoder $\mathcal{D}_{B^{t} B_{1} \rightarrow A_{0}}$ have a fidelity of at least $1-\delta$ for $\delta>0$ (cf. Theorem 14). Now, we choose $m=\lfloor\sqrt{n}\rfloor$ and $t=n-\lfloor\sqrt{n}\rfloor L$. For $n \rightarrow \infty$ the total error in (59) then tends to $1-\delta$. Moreover, we find by Lemma 15 that for $n \rightarrow \infty$ we can transmit entanglement at any rate $R \leq \inf _{i \in I} Q_{E}\left(\mathcal{N}_{A \rightarrow B}^{i}\right)$ :

$$
\frac{1}{n} \log M_{0} \geq \frac{t \cdot R}{n}=\frac{n \cdot R-\lfloor\sqrt{n}\rfloor L R}{n} \rightarrow R .
$$

Since $\delta>0$ was arbitrary, this concludes the proof.

\section{CONCLUSION}

In this article, we have determined the entanglementassisted quantum capacity of compound quantum channels for various setups. In particular, we have provided closed formulas for the entanglement-assisted capacity for uninformed users, for an informed receiver, for an informed sender, and in the presence of free feedback from the receiver to the sender. All our findings are in complete analogy to the case of classical compound channels and hence strengthen the interpretation of entanglement-assisted communication as the most tractable generalization of classical information theory to the quantum setting.

Our proofs are based on one-shot decoupling theorems, properties of smooth entropies, and make use of some previously developed tools for analyzing compound quantum channels [18], [19], [20], [24], [25], [21], [22], [23]. We believe that our approach may also lead to an improved understanding of capacities of compound quantum channels in general. As an illustration we present in Section A a simplified argument for studying the plain quantum capacity of an arbitrary compound quantum channel with an informed sender (cf. the original works [21], [22], [23]).

We end with a discussion of a few open questions. In the feedback-assisted scenario, we were only able to determine

\footnotetext{
${ }^{9}$ Since both the sender and the receiver know the classical outcome $i \in I$ we could alternatively simply use an encoder and decoder obtained by coding theorems for fixed channels (see, e.g., [30, Theorem 3.14, cf. Theorem 3.15]).
}

the capacity of finite compound quantum channels. It is not known how to extend this to arbitrary compounds and the corresponding solution for classical compounds might serve as a good starting point [8]. Moreover, it would be interesting for all the setups discussed in our work to optimize the amount of entanglement assistance that is needed, and with that to characterize the whole rate region $\left(M_{0}, M_{1}\right)$. Finally, a variant of compound quantum channels known as arbitrarily varying quantum channels (AVC) have been studied in the literature (see, e.g., [47], [48], [20], [49]). Here, for a fixed set of channels $\left\{\mathcal{N}^{i}\right\}_{i \in I}$ the goal is to find protocols for information transmission that work reliable for all channels of the form

$$
\mathcal{N}^{\left(i_{1}, \ldots, i_{n}\right)}:=\otimes_{j=1}^{n} \mathcal{N}^{i_{j}} \quad \text { in the limit } n \rightarrow \infty .
$$

The resulting entanglement-assisted arbitrarily varying quantum capacity is not known, but by analogy with the classical results [50], [51], [52] one naturally conjectures the following closed formula:

$$
Q_{E}\left(\left\{\mathcal{N}^{i}\right\}_{i \in I}\right)=\inf _{\mathcal{N} \in \operatorname{conv}\left(\left\{\mathcal{N}^{i}\right\}_{i \in I}\right)} Q_{E}(\mathcal{N})
$$

We expect the methods developed in this article to be useful for attacking the conjecture.

Note added: After completion of our work the conjecture in (60) was proven in [37].

\section{ACKNOWLEDGMENTS}

We thank David Ding, Patrick Hayden, Joseph Renes, and Volkher Scholz for helpful discussions, and Mark Wilde for pointing out the tight continuity estimate (10) (see also [39]).

\section{APPENDIX}

Proof of Lemma 5. Let $\rho_{A B}^{\prime}:=\rho_{A B} / \operatorname{tr}\left[\rho_{A B}\right]$ denote the normalized version of $\rho_{A B}$. Then, we have

$$
\begin{aligned}
& \left\|\rho_{A}^{\prime}-\rho_{A}\right\|_{1}=\left\|\rho_{A B}^{\prime}-\rho_{A B}\right\|_{1}=\left|1-\operatorname{tr}\left[\rho_{A}\right]\right| \\
= & \left|\operatorname{tr}\left[\rho_{A}-\sigma_{A}\right]\right| \leq\left\|\rho_{A}-\sigma_{A}\right\|_{1} \leq \delta,
\end{aligned}
$$

and thus $\left\|\rho_{A}^{\prime}-\sigma_{A}\right\|_{1} \leq 2 \delta$. From the first inequality in (14), we obtain that $F\left(\rho_{A}^{\prime}, \sigma_{A}\right) \geq(1-\delta)^{2}$. Thus, by Uhlmann's theorem (13) there exist partial isometries $V_{B \rightarrow C}$ and $W_{C \rightarrow B}$ such that

$$
\begin{aligned}
& F\left(V_{B \rightarrow C} \rho_{A B}^{\prime} V_{B \rightarrow C}^{\dagger}, \sigma_{A C}\right)=F\left(\rho_{A B}^{\prime}, W_{C \rightarrow B} \sigma_{A C} W_{C \rightarrow B}^{\dagger}\right) \\
\geq & (1-\delta)^{2} \geq 1-2 \delta .
\end{aligned}
$$

From the second inequality in (14), we thus obtain that $\left\|V_{B \rightarrow C} \rho_{A B}^{\prime} V_{B \rightarrow C}^{\dagger}-\sigma_{A C}\right\|_{1} \leq 2 \sqrt{2 \delta}$ and hence

$$
\begin{aligned}
& \left\|V_{B \rightarrow C} \rho_{A B} V_{B \rightarrow C}^{\dagger}-\sigma_{A C}\right\|_{1} \\
\leq & \left\|\rho_{A B}^{\prime}-\rho_{A B}\right\|_{1}+2 \sqrt{2 \delta} \leq \delta+2 \sqrt{2 \delta}
\end{aligned}
$$

Similarly, $\left\|\rho_{A B}^{\prime}-W_{C \rightarrow B} \sigma_{A C} W_{C \rightarrow B}^{\dagger}\right\|_{1} \leq 2 \sqrt{2 \delta}$ and thus

$$
\left\|\rho_{A B}-W_{C \rightarrow B} \sigma_{A C} W_{C \rightarrow B}^{\dagger}\right\|_{1} \leq \delta+2 \sqrt{2} \delta .
$$

Proof of Lemma 6. According to (21)-(22), we can find subnormalized states $\widetilde{\rho}_{A B}^{i} \in \mathcal{S}_{\leq}(A B)$ as well as $\sigma_{B}^{i} \in \mathcal{P}(B)$ such that $P\left(\rho^{i}, \widetilde{\rho}^{i}\right) \leq \varepsilon, \widetilde{\rho}_{A B}^{i} \leq I_{A} \otimes \sigma_{B}^{i}$, and $2^{-H_{\min }^{\varepsilon}(A \mid B)_{\rho^{i}}}=$ 
$\operatorname{tr}\left[\sigma_{B}^{i}\right]$ for all $i=1, \ldots, N$. Now, we define the subnormalized state $\widetilde{\rho}_{A B}:=\sum_{i=1}^{N} p_{i} \widetilde{\rho}_{A B}^{i}$. The joint quasiconvexity of the purified distance [33, (3.60)] implies that $P\left(\widetilde{\rho}_{A B}, \rho_{A B}\right) \leq \varepsilon$, and so (22) shows that

$$
H_{\min }^{\varepsilon}(A \mid B)_{\rho} \geq H_{\min }(A \mid B)_{\widetilde{\rho}} .
$$

In order to lower-bound the right-hand side, we consider $\sigma_{B}:=\sum_{i=1}^{N} p_{i} \sigma_{B}^{i}$. Clearly, $\widetilde{\rho}_{A B}=\sum_{i=1}^{N} p_{i} \widetilde{\rho}_{A B}^{i} \leq$ $\sum_{i=1}^{N} p_{i} I_{A} \otimes \sigma_{B}^{i}=I_{A} \otimes \sigma_{B}$. Thus, $\sigma_{B}$ is a feasible point for the optimization in (21), and so

$$
\begin{aligned}
& H_{\min }(A \mid B)_{\tilde{\rho}} \geq-\log \operatorname{tr}\left[\sigma_{B}\right]=-\log \left(\sum_{i=1}^{N} p_{i} \operatorname{tr}\left[\sigma_{B}^{i}\right]\right) \\
\geq & \min _{i}-\log \operatorname{tr}\left[\sigma_{B}^{i}\right]=\min _{i} H_{\min }^{\varepsilon}(A \mid B)_{\rho^{i}},
\end{aligned}
$$

using the quasi-concavity of $x \mapsto-\log x$.

Proof of Lemma 7. For all $i=1, \ldots, N$, let $\left|\rho_{A B C}^{i}\right\rangle$ a purification of $\rho_{A B}^{i}$. Then, we have that

$$
\left|\rho_{A B C I_{1} I_{2}}\right\rangle:=\frac{1}{\sqrt{N}} \sum_{i=1}^{N}\left|\rho_{A B C}^{i}\right\rangle \otimes\left|i_{I_{1}}\right\rangle\left|i_{I_{2}}\right\rangle
$$

is a purification of $\rho_{A B}$. We note that both $\rho_{A B I_{1}}$ and $\rho_{A C I_{2}}$ are classical on $I_{1}$ and $I_{2}$, respectively. Thus, we can apply [33, Lemma 6.8], which, together with (23) to switch from maxto min-entropy, shows that

$$
\begin{aligned}
H_{\max }^{\varepsilon}(A \mid B)_{\rho} & \leq H_{\max }^{\varepsilon}\left(A \mid B I_{1}\right)_{\rho}+\log N \\
& =-H_{\min }^{\varepsilon}\left(A \mid C I_{2}\right)_{\rho}+\log N \\
& \leq-\left(H_{\min }^{\varepsilon}(A \mid C)_{\rho}-\log N\right)+\log N \\
& =-H_{\min }^{\varepsilon}(A \mid C)_{\rho}+2 \log N .
\end{aligned}
$$

Now, observe that $\rho_{A C}=\frac{1}{N} \sum_{i} \rho_{A C}^{i}$. Thus, Lemma 6 can be applied, and we obtain

$$
\begin{aligned}
& -H_{\min }^{\varepsilon}(A \mid C)_{\rho} \leq-\min _{i} H_{\min }^{\varepsilon}(A \mid C)_{\rho^{i}} \\
= & \max _{i}-H_{\min }^{\varepsilon}(A \mid C)_{\rho^{i}}=\max _{i} H_{\max }^{\varepsilon}(A \mid B)_{\rho^{i}}
\end{aligned}
$$

by another application of (23).

Lemma 20. Let $\Pi_{A \rightarrow B}=\left\{\mathcal{N}_{A \rightarrow B}^{i}\right\}_{i=1}^{N}$ be a finite compound channel, $\left\{\rho_{A A^{\prime}}^{i}\right\}_{i=1}^{N}$ pure states, $M_{0}$ an integer such that $M_{0} \leq d_{A}$, and $\varepsilon \in(0,1]$. Then, there exist quantum operations $\mathcal{E}_{A_{0} \rightarrow A}^{i}$ and $\mathcal{D}_{B \rightarrow A_{0}}$ with $d_{A_{0}}=M_{0}$, such that

$$
\begin{aligned}
& \min _{i} F\left(\Phi_{A_{0} R}^{+}, \mathcal{D} \circ \mathcal{N}^{i} \circ \mathcal{E}^{i}\left(\Phi_{A_{0} R}^{+}\right)\right) \\
\geq & 1-16 N(N+2)(\sqrt{\delta}+6 \sqrt{\varepsilon}),
\end{aligned}
$$

where

$$
\delta=\max _{i} 2^{-\frac{1}{2}\left(-H_{\max }^{\varepsilon}\left(A^{\prime} \mid B\right)_{\mathcal{N}^{i}\left(\rho^{i}\right)}-\log M_{0}-2 \log N\right)} .
$$

By standard arguments this one-shot coding result can be lifted to an asymptotic IID capacity formula in terms of the regularized coherent information (as first derived and shown optimal for compound quantum channels in [21], [22], [23]).
Proof of Lemma 20. Denote by $W_{A \rightarrow B E}^{i}$ a Stinespring dilation of $\mathcal{N}_{A \rightarrow B}^{i}$ and by $\mathcal{N}_{A \rightarrow E}^{i, c}$ the corresponding complementary channel. We define the new channel

$$
\mathcal{T}_{A I \rightarrow B}(\cdot):=\sum_{i=1}^{N} \mathcal{N}_{A \rightarrow B}^{i}[\langle i|\cdot| i\rangle],
$$

and input $\rho_{A I}:=\frac{1}{N} \sum_{i} \rho_{A}^{i} \otimes|i\rangle\langle i|$ with purification $\left|\rho_{A I A^{\prime} I^{\prime}}\right\rangle:=\frac{1}{\sqrt{N}} \sum_{i}\left|\rho_{A A^{\prime}}\right\rangle \otimes|i i\rangle$. We find $\mathcal{T}_{A I \rightarrow B}\left[\rho_{A I}\right]=$ $\frac{1}{N} \sum_{i} \mathcal{N}_{A \rightarrow B}^{i}\left[\rho_{A}^{i}\right]$. We obtain a Stinespring dilation of the channel $\mathcal{T}_{A I \rightarrow B}$ by

$$
W_{A I \rightarrow B E I}(\cdot):=\sum_{i}|i\rangle\langle i| \otimes W_{A \rightarrow B E}^{i}(\cdot) .
$$

Moreover, we define the completely positive map

$$
\begin{gathered}
\mathcal{E}_{A_{0} I \rightarrow A I}\left(\sigma_{A_{0} I}\right):=d_{A I} O_{A I}(\rho) U_{A I} J_{A_{0} \rightarrow A} \\
\sigma_{A_{0} I}\left(J_{A_{0} \rightarrow A}\right)^{\dagger}\left(U_{A I}\right)^{\dagger}\left(O_{A I}(\rho)\right)^{\dagger},
\end{gathered}
$$

where the $J_{A_{0} \rightarrow A}$ are isometries, the $U_{A I}$ denote unitaries that will later be chosen at random, and $O_{A I}(\cdot)$ is defined as in (37). The main idea is to apply the one-shot decoupling lemma from [31, Theorem 3.1],

$$
\begin{aligned}
& \mathbb{E} \| \operatorname{tr}_{B}\left[W_{A I \rightarrow B E I} \mathcal{E}_{A_{0} I \rightarrow A I}\left(\Phi_{A_{0} R}^{+} \otimes|0\rangle\left\langle\left. 0\right|_{I}\right) W_{A I \rightarrow B E I}^{\dagger}\right]\right. \\
& -\omega_{E I} \otimes \tau_{R} \|_{1} \\
\leq & 2^{-\frac{1}{2}\left(H_{\min }^{\varepsilon}(A I \mid R)_{\sigma}-H_{\max }^{\varepsilon}\left(A^{\prime} I^{\prime} \mid B\right)_{\mathcal{T}(\rho)}\right)}+12 \varepsilon,
\end{aligned}
$$

where $\omega_{E I}:=\operatorname{tr}_{B}\left[W_{A I \rightarrow B E I} \rho_{A I} W_{A I \rightarrow B E I}^{\dagger}\right]$ and $\sigma_{A I R}:=$ $J_{A_{0} \rightarrow A}\left(\Phi_{A_{0} R}^{+} \otimes|0\rangle\left\langle\left. 0\right|_{I}\right) J_{A_{0} \rightarrow A}^{\dagger}\right.$. This can be simplified using the fact that $H_{\min }^{\varepsilon}(A I \mid R)_{\sigma}=-\log M_{0}$. Next, we write $\eta_{A^{\prime} I^{\prime} B}:=\frac{1}{N} \sum_{i} \mathcal{N}_{A \rightarrow B}^{i}\left(\rho_{A A^{\prime}}^{i}\right) \otimes|i\rangle\langle i|$ and apply Lemma 7 to carry out the following sequence of inequalities:

$$
\begin{aligned}
-H_{\max }^{\varepsilon}\left(A^{\prime} I^{\prime} \mid B\right)_{\eta} & \geq-H_{\max }^{\varepsilon}\left(A^{\prime} \mid B\right)_{\eta} \\
& \geq-\max _{i} H_{\max }^{\varepsilon}\left(A^{\prime} \mid B\right)_{\mathcal{N}^{i}\left(\rho^{i}\right)}-2 \log N .
\end{aligned}
$$

This implies that

$$
\begin{aligned}
& \mathbb{E} \| \operatorname{tr}_{B}\left[W_{A I \rightarrow B E I} \mathcal{E}_{A_{0} I \rightarrow A I}\left(\Phi_{A_{0} R}^{+} \otimes|0\rangle\left\langle\left. 0\right|_{I}\right) W_{A I \rightarrow B E I}^{\dagger}\right]\right. \\
& \quad-\omega_{E I} \otimes \tau_{R} \|_{1} \leq \delta+12 \varepsilon .
\end{aligned}
$$

Now Lemma 5 implies that there exists a quantum operation $\mathcal{D}_{B \rightarrow A_{0}}$ (partial isometry) such that

$$
\begin{aligned}
& \mathbb{E}\left\|\mathcal { D } _ { B \rightarrow A _ { 0 } } \circ \mathcal { T } _ { A I \rightarrow B } \circ \mathcal { E } _ { A _ { 0 } I \rightarrow A I } \left(\Phi_{A_{0} R}^{+} \otimes|0\rangle\left\langle\left. 0\right|_{I}\right)-\Phi_{A_{0} R}^{+} \|_{1}\right.\right. \\
& \leq \delta+12 \varepsilon+2 \sqrt{2(\delta+12 \varepsilon)} .
\end{aligned}
$$

On the other hand, we have

$$
\begin{aligned}
& \mathcal{T}_{A I \rightarrow B} \circ \mathcal{E}_{A_{0} I \rightarrow A I}\left(\Phi_{A_{0} R}^{+} \otimes|0\rangle\left\langle\left. 0\right|_{I}\right)\right. \\
= & \frac{1}{N} \sum_{i} \mathcal{N}_{A \rightarrow B}^{i} \circ \widetilde{\mathcal{E}}_{A_{0} \rightarrow A}^{i}\left(\Phi_{A_{0} R}^{+}\right),
\end{aligned}
$$

where we abbreviated

$$
\begin{gathered}
\widetilde{\mathcal{E}}_{A_{0} \rightarrow A}^{i}\left(\sigma_{A_{0}}\right):=d_{A} O_{A}\left(\rho^{i}\right) \sqrt{N}\left\langle i\left|U_{A I}\right| 0\right\rangle J_{A_{0} \rightarrow A} \sigma_{A_{0}} \\
\left(J_{A_{0} \rightarrow A}\right)^{\dagger} \sqrt{N}\left\langle 0\left|U_{A I}^{\dagger}\right| i\right\rangle O_{A}\left(\rho^{i}\right)^{\dagger} .
\end{gathered}
$$


Now, the one-shot decoupling lemma from [31, Theorem 3.1] gives

$$
\begin{gathered}
\quad \mathbb{E}\left\|\operatorname{tr}_{A}\left[\widetilde{\mathcal{E}}_{A_{0} \rightarrow A}^{i}\left(\Phi_{A_{0} R}^{+}\right)\right]-\tau_{R}\right\|_{1} \\
\leq 2^{-\frac{1}{2}\left(H_{\min }^{\varepsilon}\left(A^{\prime} I^{\prime}\right)_{\rho^{i}}-\log M_{0}\right)}+12 \varepsilon,
\end{gathered}
$$

where we have introduced $\rho_{A^{\prime} I^{\prime}}^{i}=\rho_{A^{\prime}}^{i} \otimes|i\rangle\left\langle\left. i\right|_{I^{\prime}}\right.$. We now note that

$$
\begin{aligned}
H_{\min }^{\varepsilon}\left(A^{\prime} I^{\prime}\right)_{\rho^{i}} & =H_{\min }^{\varepsilon}\left(A^{\prime}\right)_{\rho^{i}} \\
& \geq H_{\min }^{\varepsilon}\left(A^{\prime} \mid E\right)_{\mathcal{N}^{i, c}\left(\rho^{i}\right)}=-H_{\max }^{\varepsilon}\left(A^{\prime} \mid B\right)_{\mathcal{N}^{i}\left(\rho^{i}\right)}
\end{aligned}
$$

which implies that

$$
\mathbb{E}\left\|\operatorname{tr}_{A}\left[\widetilde{\mathcal{E}}_{A_{0} \rightarrow A}^{i}\left(\Phi_{A_{0} R}^{+}\right)\right]-\tau_{R}\right\|_{1} \leq \delta+12 \varepsilon .
$$

Combining (61), (62), Markov's inequality $\operatorname{Prob}(Z>$ $k \mathbb{E}(Z)) \leq 1 / k$ (for $k=N+2$ ), and the union bound, we find that there exist a unitary $U_{A I}$ and a quantum operation $\mathcal{D}_{B \rightarrow A_{0}}$ such that

$$
\left\|\operatorname{tr}_{A}\left[\widetilde{\mathcal{E}}_{A_{0} \rightarrow A}^{i}\left(\Phi_{A_{0} R}^{+}\right)\right]-\tau_{R}\right\|_{1} \leq(N+2)(\delta+12 \varepsilon)
$$

for $i=1, \ldots, N$, with

$$
\begin{aligned}
& \left\|\mathcal{D}_{B \rightarrow A_{0}} \circ \frac{1}{N} \sum_{i=1}^{N} \mathcal{N}_{A \rightarrow B}^{i} \circ \widetilde{\mathcal{E}}_{A_{0} \rightarrow A}^{i}\left(\Phi_{A_{0} R}^{+}\right)-\Phi_{A_{0} R}^{+}\right\|_{1} \\
& \leq(N+2)(\delta+12 \varepsilon+2 \sqrt{2(\delta+12 \varepsilon})) .
\end{aligned}
$$

As a consequence of the first bound and Lemma 5, we can then find quantum operations $\left\{\mathcal{E}_{A_{0} \rightarrow A}^{i}\right\}_{i=1}^{N}$ (partial isometries) such that

$$
\begin{aligned}
& \left\|\left(\widetilde{\mathcal{E}}_{A_{0} \rightarrow A}^{i}-\mathcal{E}_{A_{0} \rightarrow A}^{i}\right)\left(\Phi_{A_{0} R}^{+}\right)\right\|_{1} \\
\leq & (N+2)(\delta+12 \varepsilon)+2 \sqrt{2(N+2)(\delta+12 \varepsilon)} .
\end{aligned}
$$

Using the triangle inequality as well as the fact that $\mathcal{D}$ and the $\mathcal{N}^{i}$ are completely positive and trace-nonincreasing, we obtain that

$$
\begin{aligned}
& \left\|\left(\mathcal{D}_{B \rightarrow A_{0}} \circ \frac{1}{N} \sum_{i=1}^{N} \mathcal{N}_{A \rightarrow B}^{i} \circ \mathcal{E}_{A_{0} \rightarrow A}^{i}\right)\left(\Phi_{A_{0} R}^{+}\right)-\Phi_{A_{0} R}^{+}\right\|_{1} \\
\leq & \left\|\left(\mathcal{D}_{B \rightarrow A_{0}} \circ \frac{1}{N} \sum_{i=1}^{N} \mathcal{N}_{A \rightarrow B}^{i} \circ \widetilde{\mathcal{E}}_{A_{0} A_{1} \rightarrow A}^{i}\right)\left(\Phi_{A_{0} R}^{+}\right)-\Phi_{A_{0} R}^{+}\right\|_{1} \\
& +\frac{1}{N} \sum_{i=1}^{N}\left\|\left(\widetilde{\mathcal{E}}_{A_{0} \rightarrow A}^{i}-\mathcal{E}_{A_{0} \rightarrow A}^{i}\right)\left(\Phi_{A_{0} R}^{+}\right)\right\|_{1} \\
\leq & (N+2)(\delta+12 \varepsilon)+2 \sqrt{2(N+2)(\delta+12 \varepsilon)} \\
& +(N+2)(\delta+12 \varepsilon+2 \sqrt{2(\delta+12 \varepsilon})) \\
\leq & 8(N+2)(\sqrt{\delta}+6 \sqrt{\varepsilon}) .
\end{aligned}
$$

For the last step we assumed that $\delta \in(0,1]$ (without loss of generality). Lastly, we use (15) to turn this into a lower bound on the entanglement fidelity:

$$
\begin{aligned}
& F\left(\Phi_{A_{0} R}^{+},\left(\frac{1}{N} \sum_{i=1}^{N} \mathcal{D}_{B \rightarrow A_{0}} \circ \mathcal{N}_{A \rightarrow B}^{i} \circ \mathcal{E}_{A_{0} \rightarrow A}^{i}\right)\left(\Phi_{A_{0} R}^{+}\right)\right) \\
\geq & 1-16(N+2)(\sqrt{\delta}+6 \sqrt{\varepsilon}) .
\end{aligned}
$$

Using the same argument that was used to derive (29) this implies the claim.

\section{REFERENCES}

[1] C. Shannon, "A mathematical theory of communication," Bell System Technical Journal, vol. 27, pp. 379-423, 1948.

[2] C. H. Bennett, P. W. Shor, J. A. Smolin, and A. Thapliyal, "Entanglement-assisted capacity of a quantum channel and the reverse Shannon theorem," IEEE Transactions on Information Theory, vol. 48, no. 10, pp. 2637-2655, 2002.

[3] W. Matthews and S. Wehner, "Finite blocklength converse bounds for quantum channels," IEEE Transactions on Information Theory, vol. 60, no. 11, pp. 7317-7329, 2014

[4] A. Lapidoth and P. Narayan, "Reliable communication under channel uncertainty," IEEE Transactions on Information Theory, vol. 44, no. 6, pp. 2148-2177, 1998.

[5] D. Blackwell, L. Breiman, and A. J. Thomasian, "The capacity of a class of channels," Annals of Mathematical Statistics, vol. 30, no. 4, pp. $1229-1241,1959$.

[6] J. Wolfowitz, "Simultaneous channels," Archive for Rational Mechanics and Analysis, vol. 4, no. 1, pp. 371-386, 1959.

[7] — Coding Theorems of Information Theory, 3rd ed. Springer-Verlag, New York, 1978.

[8] B. Shrader and H. Permuter, "Feedback capacity of the compound channel," IEEE Transactions on Information Theory, vol. 55, no. 8, pp. 3629-3644, 2009.

[9] A. Holevo, "The capacity of the quantum channel with general signal states," IEEE Transactions on Information Theory, vol. 44, no. 1, pp. 269-273, 1998

[10] B. Schumacher and M. Westmoreland, "Sending classical information via noisy quantum channels," Physical Review A, vol. 56, no. 1, pp. 131-138, 1997.

[11] I. Devetak, "The private classical capacity and quantum capacity of a quantum channel," IEEE Transactions on Information Theory, vol. 51, no. 1 , pp. 44-55, 2005.

[12] S. Lloyd, "The capacity of the noisy quantum channel," Physical Review A, vol. 55, no. 3, pp. 1613-1622, 1996.

[13] P. W. Shor, "The quantum channel capacity and coherent information," in Lectures Notes, MSRI Workshop on Quantum Computation, 2002.

[14] G. Bowen, "Quantum feedback channels," IEEE Transactions on Infor mation Theory, vol. 50, no. 10, pp. 2429-2434, 2004.

[15] D. Leung and W. Matthews, "On the power of ppt-preserving and nonsignalling codes," IEEE Transactions on Information Theory, vol. 61, no. 8, pp. 4486-4499, 2015.

[16] C. H. Bennett, G. Brassard, C. Crépeau, R. Jozsa, A. Peres, and W. K Wootters, "Teleporting an unknown quantum state via dual classical and Einstein-Podolsky-Rosen channels," Physical Review Letters, vol. 70, pp. 1895-1899, 1993.

[17] C. H. Bennett and S. J. Wiesner, "Communication via one- and twoparticle operators on Einstein-Podolsky-Rosen states," Physical Review Letters, vol. 69, pp. 2881-2884, 1992.

[18] I. Bjelaković and H. Boche, "Classical capacities of compound and averaged quantum channels," IEEE Transactions on Information Theory, vol. 55, no. 7, pp. 3360-3374, 2009.

[19] M. Hayashi, "Universal coding for classical-quantum channel," Communications in Mathematical Physics, vol. 289, no. 3, pp. 1087-1098, 2009.

[20] I. Bjelaković, H. Boche, G. Janßen, and J. Nötzel, "Arbitrarily varying and compound classical-quantum channels and a note on quantum zeroerror capacities," in Information Theory, Combinatorics, and Search Theory, ser. Lecture Notes in Computer Science Volume, vol. 7777. Springer, 2013, pp. 247-283.

[21] I. Bjelaković, H. Boche, and J. Nötzel, "Quantum capacity of a class of compound channels," Physical Review A, vol. 78, no. 4, p. 042331, 2008.

[22] I. Bjelaković, H. Boche, and J. Nötzel, "Entanglement transmission capacity of compound channels," in Proc. IEEE ISIT 2009, 2009, pp. $1889-1893$.

[23] _ - "Entanglement transmission and generation under channel uncertainty: Universal quantum channel coding," Communications in Mathematical Physics, vol. 292, no. 1, pp. 55-97, 2009.

[24] N. Datta and T. C. Dorlas, "The coding theorem for a class of quantum channels with long-term memory," Journal of Physics A: Mathematical and Theoretical, vol. 40, no. 28, p. 8147, 2007. 
[25] N. Datta, Y. Suhov, and T. C. Dorlas, "Entanglement assisted classical capacity of a class of quantum channels with long-term memory," Quantum Information Processing, vol. 7, no. 6, pp. 251-262, 2008.

[26] M. Horodecki, J. Oppenheim, and A. Winter, "Partial quantum information," Nature, vol. 436, no. 7051, pp. 673-6, 2005.

[27] — "Quantum state merging and negative information," Communications in Mathematical Physics, vol. 269, no. 1, pp. 107-136, 2006.

[28] P. Hayden, M. Horodecki, J. Yard, and A. Winter, "A decoupling approach to the quantum capacity," Open Systems \& Information Dynamics, vol. 15 , no. 01 , p. 7, 2008.

[29] A. Abeyesinghe, I. Devetak, P. Hayden, and A. Winter, "The mother of all protocols: Restructuring quantum information's family tree," Proceedings of the Royal Society A, vol. 465, no. 2108, pp. 2537-2563, 2009.

[30] F. Dupuis, "The decoupling approach to quantum information theory," Ph.D. dissertation, Université de Montréal, 2009.

[31] F. Dupuis, M. Berta, J. Wullschleger, and R. Renner, "One-shot decoupling," Communications in Mathematical Physics, vol. 328, no. 1, pp. 251-284, 2014

[32] R. Renner, "Security of quantum key distribution," Ph.D. dissertation, ETH Zurich, 2005.

[33] M. Tomamichel, Quantum Information Processing with Finite Resources. Springer, 2016.

[34] D. Kretschmann and R. F. Werner, "Tema con variazioni: quantum channel capacity," New Journal of Physics, vol. 6, no. 1, p. 26, 2004.

[35] R. Alicki and M. Fannes, "Continuity of quantum conditional information," Journal of Physics A: Mathematical and General, vol. 37, no. 5, p. L55, 2004.

[36] A. Winter, "Tight uniform continuity bounds for quantum entropies: Conditional entropy, relative entropy distance and energy constraints," Communications in Mathematical Physics, vol. 347, no. 1, pp. 291-313, 2016.

[37] H. Boche, G. Janssen, and S. Kaltenstadler, "Entanglement-assisted classical capacities of compound and arbitrarily varying quantum channels," Quantum Information Processing (to appear), 2017.

[38] , "Entanglement assisted classical capacity of compound quantum channels," in Proc. IEEE ISIT 2016, 2016, pp. 1680-1684.

[39] M. M. Wilde, Quantum Information Theory. Cambridge University Press, 2013.

[40] O. Fawzi and R. Renner, "Quantum conditional mutual information and approximate Markov chains," Communications in Mathematical Physics, vol. 340, no. 2, pp. 575-611, 2015.

[41] M. Shirokov, "Tight continuity bounds for the quantum conditional mutual information, for the Holevo quantity and for capacities of quantum channels," 2015.

[42] M. Tomamichel, R. Colbeck, and R. Renner, "Duality between smooth min- and max-entropies," IEEE Transactions on Information Theory, vol. 56, no. 9, pp. 4674-4681, 2010.

[43] R. König, R. Renner, and C. Schaffner, "The operational meaning of minand max-entropy," IEEE Transactions on Information Theory, vol. 55, no. 9, pp. 4337-4347, 2009.

[44] M. Tomamichel, R. Colbeck, and R. Renner, "A fully quantum asymptotic equipartition property," IEEE Transactions on Information Theory, vol. 55, no. 12, pp. 5840-5847, 2009.

[45] C. Adami and N. Cerf, "Von Neumann capacity of noisy quantum channels," Physical Review A, vol. 56, no. 5, pp. 3470-3483, 1997.

[46] R. Bhatia, Matrix analysis. Springer-Verlag New York, 2013.

[47] R. Ahlswede, I. Bjelaković, H. Boche, and J. Nötzel, "Entanglement transmission over arbitrarily varying quantum channels," in Proc. IEEE ISIT 2010, 2010, pp. 2718-2722.

[48] R. Ahlswede, I. Bjelaković, H. Boche, and J. Nötzel, "Quantum capacity under adversarial quantum noise: Arbitrarily varying quantum channels," Communications in Mathematical Physics, vol. 317, no. 1, pp. 103-156, 2012.

[49] H. Boche and J. Nötzel, "Arbitrarily small amounts of correlation for arbitrarily varying quantum channels," Journal of Mathematical Physics, vol. 54, no. 11, p. 112202, 2013.

[50] D. Blackwell, L. Breiman, and A. J. Thomasian, "The capacities of certain channel classes under random coding," Annals of Mathematical Statistics, vol. 31, pp. 558-567, 1960.

[51] I. G. Stiglitz, "Coding for a class of unknown channels," IEEE Transactions on Information Theory, vol. 12, pp. 189-195, 1966.

[52] R. Ahlswede and J. Wolfowitz, "Correlated decoding for channels with arbitrarily varying channel probability functions," Information and Control, vol. 14, pp. 457-473, 1969. 